\title{
Mining Closed Strict Episodes ${ }^{\star}$
}

\author{
Nikolaj Tatti and Boris Cule
}

the date of receipt and acceptance should be inserted later

\begin{abstract}
Discovering patterns in a sequence is an important aspect of data mining. One popular choice of such patterns are episodes, patterns in sequential data describing events that often occur in the vicinity of each other. Episodes also enforce in which order the events are allowed to occur.

In this work we introduce a technique for discovering closed episodes. Adopting existing approaches for discovering traditional patterns, such as closed itemsets, to episodes is not straightforward. First of all, we cannot define a unique closure based on frequency because an episode may have several closed superepisodes. Moreover, to define a closedness concept for episodes we need a subset relationship between episodes, which is not trivial to define.

We approach these problems by introducing strict episodes. We argue that this class is general enough, and at the same time we are able to define a natural subset relationship within it and use it efficiently. In order to mine closed episodes we define an auxiliary closure operator. We show that this closure satisfies the needed properties so that we can use the existing framework for mining closed patterns. Discovering the true closed episodes can be done as a post-processing step. We combine these observations into an efficient mining algorithm and demonstrate empirically its performance in practice.
\end{abstract}

Keywords Frequent Episode Mining, Closed Episodes, Level-wise Algorithm

\section{Introduction}

Discovering frequent patterns in an event sequence is an important field in data mining. Episodes, as defined in [12], represent a rich class of sequential patterns, enabling us to discover events occurring in the vicinity of each other while at the same time capturing complex interactions between the events.

* A preliminary version appeared as "Mining Closed Strict Episodes", in Proceedings of Tenth IEEE International Conference on Data Mining (ICDM 2010), 2010 [17].

Nikolaj Tatti · Boris Cule

University of Antwerp, Antwerp, Belgium,

E-mail: nikolaj.tatti@ua.ac.be, boris.cule@ua.ac.be 
More specifically, a frequent episode is traditionally considered to be a set of events that reoccurs in the sequence within a window of a specified length. Gaps are allowed between the events and the order in which the events are allowed to occur is specified by the episode. Frequency, the number of windows in which the episode occurs, is monotonically decreasing so we can use the well-known level-wise approach to mine all frequent episodes.

The order restrictions of an episode are described by a directed acyclic graph (DAG): the set of events in a sequence covers the episode if and only if each event occurs only after all its parent events (with respect to the DAG) have occurred (see the formal definition in Section 2). Usually, only two extreme cases are considered. A parallel episode poses no restrictions on the order of events, and a window covers the episode if the events occur in the window, in any order. In such a case, the DAG associated with the episode contains no edges. The other extreme case is a serial episode. Such an episode requires that the events occur in one, and only one, specific order in the sequence. Clearly, serial episodes are more restrictive than parallel episodes. If a serial episode is frequent, then its parallel version is also frequent.

The advantage of episodes based on DAGs is that they allow us to capture dependencies between the events while not being too restrictive.

Example 1 As an example we will use text data, namely inaugural speeches by presidents of the United States (see Section 8 for more details). Protocol requires the presidents to address the chief justice and the vice presidents in their speeches. Hence, a pattern

$$
\text { chief } \rightarrow \text { justic } \quad \text { vice } \rightarrow \text { president }
$$

occurs in 10 disjoint windows. This pattern captures the phrases 'chief justice' and 'vice president' but because the address order varies from speech to speech, the pattern does not impose any additional restrictions.

Episodes based on DAGs have, in practice, been over-shadowed by parallel and serial episodes, despite being defined at the same time [12]. The main reason for this is the pattern explosion demonstrated in the following example.

Example 2 To illustrate the pattern explosion we will again use inaugural speeches by presidents of the United States. By setting the window size to 15 and the frequency threshold to 60 we discovered a serial episode with 6 symbols,

$$
\text { preserv } \rightarrow \text { protect } \rightarrow \text { defend } \rightarrow \text { constitut } \rightarrow \text { unit } \rightarrow \text { state. }
$$

In total, we found another 4823 subepisodes of size 6 of this episode. However, all these episodes had only 3 distinct frequencies, indicating that the frequencies of most of them could be derived from the frequencies of only 3 episodes, so the output could be reduced by leaving out 4821 episodes.

We illustrate the pattern explosion further in Table 1 . We see from the table that if the sequence has a frequent serial episode consisting of 9 labels, then mining frequent episodes will produce at least 100 million patterns.

Motivated by this example, we approach the problem of pattern explosion by using a popular technique of closed patterns. A pattern is closed if there exists no superpattern with the same frequency. Mining closed patterns has been shown to reduce the output. Moreover, we can discover closed patterns efficiently. However, adopting the concept of closedness to episodes is not without problems. 


\begin{tabular}{lrrrrrrrrr}
\hline pattern & 1 & 2 & 3 & 4 & 5 & 6 & 7 & 8 & 9 \\
\hline itemsets & 1 & 3 & 7 & 15 & 31 & 63 & 127 & 255 & 511 \\
episodes & 1 & 4 & 16 & 84 & 652 & 7742 & 139387 & 3730216 & 145605024 \\
\hline
\end{tabular}

Table 1 Illustration of the pattern explosion. The first row is the number of frequent itemsets produced by a single frequent itemset with $n$ items. The second row is the number of episodes produced by a single frequent serial episode with $n$ unique labels. These numbers were obtained by a brute force enumeration.

Subset relationship Establishing a proper subset relationship is needed for two reasons. Firstly, to make the mining procedure more efficient by discovering all possible subpatterns before testing the actual episode, and secondly, to define a proper closure operator.

A naïve approach to define whether an episode $G$ is a subepisode of an episode $H$ is to compare their DAGs. This, however, leads to problems as the same episode can be represented by multiple DAGs and a graph representing $G$ is not necessarily a subgraph of a graph representing $H$ as demonstrated in the following example.

Example 3 Consider episodes $G_{1}, G_{2}$, and $G_{3}$ given in Figure 1. Episode $G_{1}$ states that for a pattern to occur $a$ must precede $b$ and $c$. Meanwhile, $G_{2}$ and $G_{3}$ state that $a$ must be followed by $b$ and then by $c$. Note that $G_{2}$ and $G_{3}$ represent essentially the same pattern that is more restricted than the pattern represented by $G_{1}$. However, $G_{1}$ is a subgraph of $G_{3}$ but not a subgraph of $G_{2}$. This reveals a problem if we base our definition of a subset relationship of episodes solely on the edge subset relationship. We solve this particular case by considering transitive closures, graphs in which each node must be connected to all its descendants by an edge, thus ignoring graphs of form $G_{2}$. We will not lose any generality since we are still going to discover episodes of form $G_{3}$. Using transitive closure does not solve all problems for episodes containing multiple nodes with the same label. For example, episodes $H_{1}$ and $H_{2}$ in Figure 1 are the same even though their graphs are different.

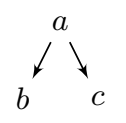

(a) Episode $G_{1}$ $a \rightarrow b \rightarrow c$

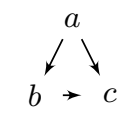

(b) Episode $G_{2}$

(c) Episode $G_{3}$

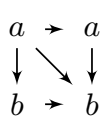

(d) Episode $H_{1}$

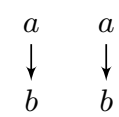

(e) Episode $\mathrm{H}_{2}$

Fig. 1 Toy episodes used in Example 3.

Frequency closure Secondly, frequency does not satisfy the Galois connection. In fact, given an episode $G$ there can be several more specific closed episodes that have the same frequency. So the closure operator cannot be defined as a mapping from an episode to its frequency-closed version.

Example 4 Consider sequence $s$ given in Figure $2(\mathrm{e})$ and episode $G_{1}$ given in Figure 2(a). Assume that we use a sliding window of size 5 . There are two windows that cover episode $G_{1}$, namely $s[1,5]$ and $s[6,10]$, illustrated in Figure $2(\mathrm{e})$. Hence, the frequency of $G_{1}$ is 2 . There are two serial episodes that are more specific than $G_{1}$ and 
have the same frequency, namely, $G_{2}$ and $G_{3}$ given in Figures 2(b) and 2(c). Moreover, there is no superepisode of $G_{2}$ and $G_{3}$ that has frequency equal to 2 . In other words, we cannot define a unique closure for $G_{1}$ based on frequency.

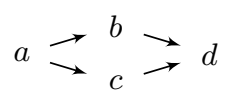

(a) Episode $G_{1}$

$$
a \rightarrow b \rightarrow c \rightarrow d \quad a \rightarrow c \rightarrow b \rightarrow d
$$

(b) Episode $G_{2}$

(c) Episode $G_{3}$

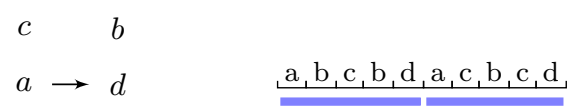

$\begin{array}{ll}\text { (d) Episode } G_{4} & \text { (e) Sequence } s\end{array}$

Fig. 2 Toy episodes used in Examples 4 and 5. Edges induced by transitive closure are omitted to avoid clutter.

The contributions of our paper address these issues:

1. We introduce strict episodes, a new subclass of general episodes. We say that an episode is strict if all nodes with the same label are connected. Thus all episodes in Figure 1 are strict, except $H_{2}$. We will argue that this class is large, contains all serial and parallel episodes, as well as episodes with unique labels, yet using only strict episodes eases the computational burden.

2. We introduce a natural subset relationship between episodes based on the subset relationship of sequences covering the episodes. We will prove that for strict episodes this relationship corresponds to the subset relationship between transitively closed graphs. For strict episodes such a graph uniquely defines the episode.

3. We introduce milder versions of the closure concept, including the instance-closure. We will show that these closures can be used efficiently, and that a frequency-closed episode is always instance-closed ${ }^{1}$. We demonstrate that computing closure and frequency can be done in polynomial time ${ }^{2}$.

4. Finally, we present an algorithm that generates strict instance-closed episodes with transitively closed graphs. Once these episodes are discovered we can further prune the output by removing the episodes that are not frequency-closed.

\section{Preliminaries and Notation}

We begin by presenting the preliminary concepts and notations that will be used throughout the paper. In this section we introduce the notions of sequence and episodes.

A sequence $s=s_{1} \cdots s_{L}$ is a string of symbols, or events, coming from an alphabet $\Sigma$, so that for each $i, s_{i} \in \Sigma$. Given a strictly increasing mapping $m:[1, N] \rightarrow[1, L]$ we will define $s_{m}$ to be a subsequence $s_{m(1)} \cdots s_{m(N)}$. Similarly, given two integers $1 \leq a \leq b \leq L$ we define $s[a, b]=s_{a} \cdots s_{b}$.

An episode $G$ is represented by a directed acyclic graph with labelled nodes, that is, $G=(V, E, l a b)$, where $V=\left(v_{1}, \ldots, v_{K}\right)$ is the set of nodes, $E$ is the set of directed

1 In [17], the closure was based only on adding edges whereas in this version we are also adding nodes.

2 This was not guaranteed in [17]. 
edges, and $l a b$ is the function $l a b: V \rightarrow \Sigma$, mapping each node $v_{i}$ to its label. We denote the set of nodes of an episode $G$ with $V(G)$, and its set of edges with $E(G)$.

Given a sequence $s$ and an episode $G$ we say that $s$ covers $G$, or $G$ occurs in $s$, if there is an injective map $f$ mapping each node $v_{i}$ to a valid index such that the node $v_{i}$ in $G$ and the corresponding sequence element $s_{f\left(v_{i}\right)}$ have the same label, $s_{f\left(v_{i}\right)}=l a b\left(v_{i}\right)$, and that if there is an edge $\left(v_{i}, v_{j}\right)$ in $G$, then we must have $f\left(v_{i}\right)<$ $f\left(v_{j}\right)$. In other words, the parents of $v_{j}$ must occur in $s$ before $v_{j}$. For an example, see Figure 3(a). If the mapping $f$ is surjective, that is, all events in $s$ are used, we will say that $s$ is an instance of $G$.

\subsection{Frequency}

In our search for frequent episodes, we will use and compare two conceptually different definitions of frequency.

Traditionally, episode mining is based on searching for episodes that are covered by windows of certain fixed size often enough. The frequency of a given episode is then defined as the number of such windows that cover it.

Definition 1 The fixed-window frequency of an episode $G$ in a sequence $s$, denoted $f r_{f}(G)$, is defined as the number of time windows of a given size $\rho$ within $s$, in which the episode $G$ occurs. Formally,

$$
f r_{f}(G)=\mid\{(a, b) \mid b=a+\rho-1, a \leq N, b \geq 1 \text {, and } s[a, b] \text { covers } G\} \mid .
$$

See Figure 3(b) for example.

The frequency of an episode is sometimes expressed using the number of minimal windows that contain it. To satisfy the downward-closed property, we say that these windows must be non-overlapping.

Definition 2 The disjoint-window frequency of an episode $G$ in a sequence $s$, denoted $f r_{d}(G)$, is defined as the maximal number of non-overlapping windows within $s$ that contain episode $G$. Formally,

$$
f r_{d}(G)=\max \left\{\begin{array}{l|l}
\left|\left\{\left(a_{1}, b_{1}\right), \ldots,\left(a_{N}, b_{N}\right)\right\}\right| \mid \begin{array}{l}
s\left[a_{i}, b_{i}\right] \text { covers } G, b_{i}-a_{i}<\rho \text { and } \\
{\left[a_{i}, b_{i}\right] \cap\left[a_{j}, b_{j}\right]=\emptyset \text { for } 1 \leq i, j \leq N}
\end{array}
\end{array}\right\}
$$

See Figure 3(c) for example.

We now establish a connection between the disjoint-window frequency and actual minimal windows.

Definition 3 Given a sequence $s$ and an episode $G$, a window $s[a, b]$ is called a minimal window of $G$ in $s$, if $s[a, b]$ covers $G$, and if no proper subwindow of $s[a, b]$ covers $G$. We will also refer to the interval $[a, b]$ as a minimal window, if $s$ is clear from the context.

It is easy to see that the maximal number of non-overlapping windows within $s$ that contain $G$ is equal to the maximal number of non-overlapping minimal windows within $s$ that contain $G$.

Whenever it does not matter whether we are dealing with the fixed-window frequency or the disjoint-window frequency, we simply denote $\operatorname{fr}(G)$. 


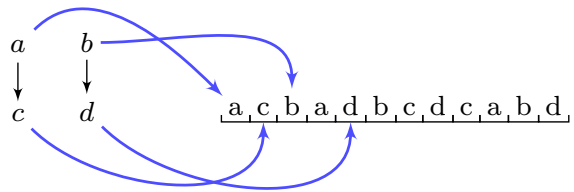

(a) An example of an episode covered by a sequence

$\mathrm{a}, \mathrm{b}, \mathrm{c}, \mathrm{a}, \mathrm{d}, \mathrm{c}, \mathrm{b}, \mathrm{d}, \mathrm{b}, \mathrm{a}, \mathrm{c}, \mathrm{d}$

(b) fixed-length windows $a, b, c, a, d, c, b, d, b, a, c, d$

(c) non-overlapping windows

Fig. 3 A toy example illustrating different support measures. Figure 3(a) contains an example of a sequence covering an episode. Figure 3(b) shows all 5 sliding windows of length 5 containing the episode. Figure $3(\mathrm{c})$ shows the maximal number, 2, of non-overlapping windows covering the episode.

\section{Strict Episodes}

In this section we will define our mining problem and give a rough outline of the discovery algorithm.

Generally, a pattern is considered closed if there exists no more specific pattern having the same frequency. In order to speak of more specific patterns, we must first have a way to describe episodes in these terms.

Definition 4 Assume two transitively closed episodes $G$ and $H$ with the same number of nodes. An episode $G$ is called a subepisode of episode $H$, denoted $G \preceq H$ if the set of all sequences that cover $H$ is a subset of the set of all sequences that cover $G$. If the set of all sequences that cover $H$ is a proper subset of the set of all sequences that cover $G$, we call $G$ a proper subepisode of $H$, denoted $G \prec H$.

For a more general case, assume that $|V(G)|<|V(H)|$. We say that $G$ is a subepisode of $H$, denoted $G \preceq H$, if there is a subgraph $H^{\prime}$ of $H$ such that $G \preceq H^{\prime}$. Moreover, let $\alpha$ be a graph homomorphism from $H^{\prime}$ to $H$. If we wish to emphasize $\alpha$, we write $G \preceq \alpha H$.

If $|V(G)|>|V(H)|$, then $G$ is automatically not a subepisode of $H$.

The problem with this definition is that we do not have the means to compute this relationship for general episodes. To do this, one would have to enumerate all possible sequences that cover $H$ and compute whether they cover $G$. We approach this problem by restricting ourselves to a class of episodes where this comparison can be performed efficiently.

Definition 5 An episode $G$ is called strict if for any two nodes $v$ and $w$ in $G$ sharing the same label, there exists a path either from $v$ to $w$ or from $w$ to $v$.

We will show later that the subset relationship can be computed efficiently for strict episodes. However, as can be seen in Figure 4, there are episodes that are not strict. Our algorithm will not discover these types of patterns.

Having defined a subset relationship, we can now define an $f$-closed episode.

Definition 6 An episode $G$ is frequency-closed, or $f$-closed, if there exists no episode $H$, such that $G \prec H$ and $\operatorname{fr}(G)=\operatorname{fr}(H)$. 


$\begin{array}{lll}a & a & a \rightarrow a \\ \downarrow & \downarrow & \downarrow \searrow \downarrow \\ b & c & b\end{array}$

$\begin{array}{ll}\text { (a) non-strict } & \text { (b) strict }\end{array}$

Fig. 4 An example of a non-strict and a strict episode.

Problem 1 Given a sequence $s$, a frequency measure, either fixed-window or disjointwindow, and a threshold $\sigma$, find all $f$-closed strict episodes from $s$ having the frequency higher or equal than $\sigma$.

A traditional approach to discovering closed patterns is to discover generators, that is, for each closed pattern $P$, discover minimal patterns whose closure is equal to $P$ [14]. When patterns are itemsets, it holds that the collection of frequent generators are downward closed. Hence, they can be mined efficiently using a BFS-style approach.

We cannot directly apply this framework for two reasons: Firstly, unlike with itemsets, we cannot define a closure based on frequency. We solve this by defining an instance-closure, a more conservative closure that guarantees that all $f$-closed episodes are discovered. Once instance-closed episodes are discovered, the $f$-closed episodes are selected in a post-processing step. The second obstacle is the fact that the collection of generator episodes is not necessarily downward-closed. We solve this problem by additionally using some intermediate episodes that will guarantee the correctness of the algorithm.

A sketch of the miner is given in Algorithm 1 (the details of the algorithm are described in subsequent sections). The algorithm consists of two loops. In the outer loop, Lines 3-15, we discover parallel episodes by adding nodes. In the inner loop, Lines $5-12$, we discover general episodes by adding edges. Each candidate episode is tested, and if the candidate is frequent and a generator, then the episodes is added into the collection. Finally, we discover the $f$-closed episodes as a last step.

To complete the algorithm we need to solve several subproblems:

1. computing the subset relationship efficiently (Section 4)

2. defining and computing instance-closure (Section 5)

3. generating candidate episodes, Line 11 (Section 6)

4. generating intermediate episodes and proving the correctness (Section 7)

\section{Computing The Subset Relationship}

In this section we will demonstrate that computing the subset relationship for strict episodes can be done efficiently. This allows us to build an algorithm to efficiently discover closed episodes.

We now provide a canonical form for episodes, which will help us in further theorems and algorithms. We define an episode that has the maximal number of edges using a fundamental notion familiar from graph theory.

Definition 7 The transitive closure of an episode $G=(V, E, l a b)$ is an episode $t c l(G)$, where $G$ and $\operatorname{tcl}(G)$ have the same set of nodes $V$, the same lab function mapping nodes to labels, and the set of edges in $\operatorname{tcl}(G)$ is equal to

$$
E(\operatorname{tcl}(G))=E \cup\left\{\left(v_{i}, v_{j}\right) \mid \text { a path exists in } G \text { from } v_{i} \text { to } v_{j}\right\} .
$$




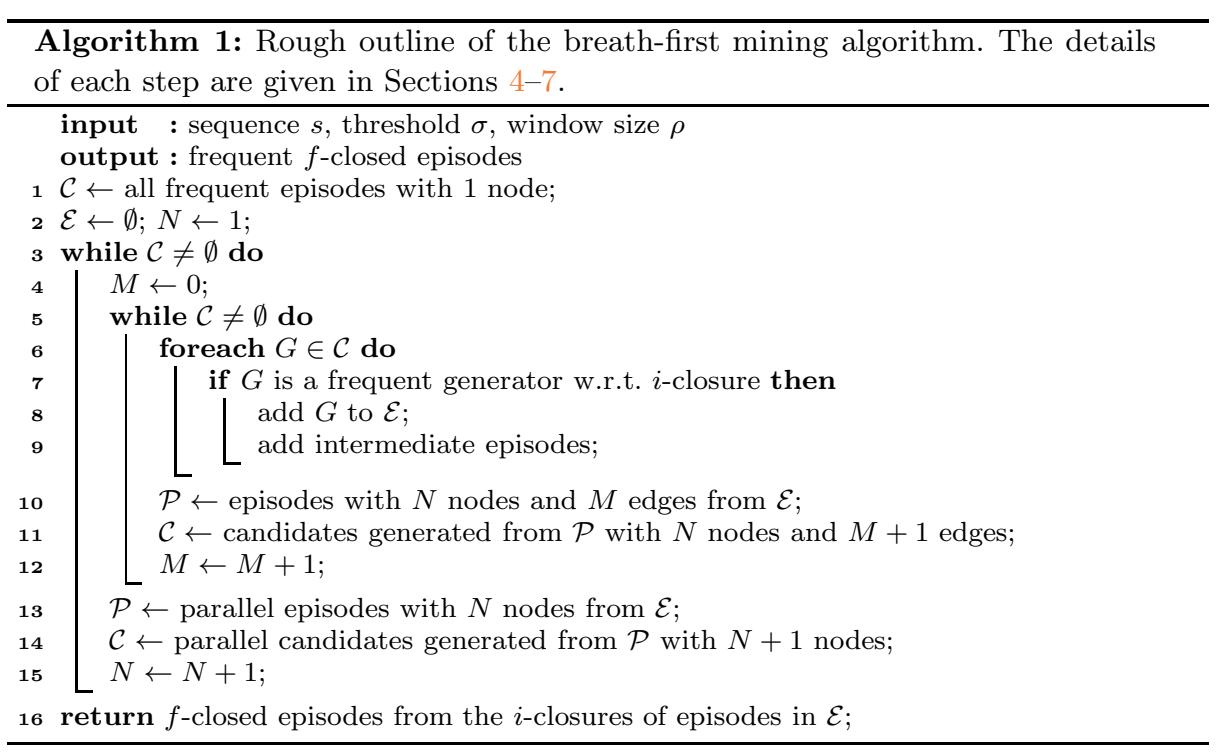

Note that, despite its name, the transitive closure has nothing to do with the concept of closed episodes.

Definition 8 Let $\mathcal{S}$ be the space of all strict and transitively closed episodes.

In the remaining text, we consider episodes to be transitively closed and strict, unless stated otherwise. An episode and its transitive closure will always have the same frequency, hence by restricting ourselves to tranistively closed episodes we will not lose any episodes.

For notational simplicity, we now introduce the concept of two episodes having identical nodes. Given an episode $G \in \mathcal{S}$ with nodes $V(G)=\left\{v_{1}, \ldots, v_{N}\right\}$, we assume from now on that the order of the nodes is always fixed such that for $i<j$ either $l a b\left(v_{i}\right)<l a b\left(v_{j}\right)$ lexicographically, or $l a b\left(v_{i}\right)=l a b\left(v_{j}\right)$ and $v_{i}$ is an ancestor of $v_{j}$ with respect to $E(G)$ (i.e. edge $\left(v_{i}, v_{j}\right) \in E(G)$ ). We say that two episodes $G$ and $H$, with $V(G)=\left\{v_{1}, \ldots, v_{N}\right\}$ and $V(H)=\left\{w_{1}, \ldots, w_{N}\right\}$ have identical nodes if $l a b\left(v_{i}\right)=l a b\left(w_{i}\right)$ for $i=\{1, \ldots, N\}$. To simplify notation, we often identify $v_{i}$ and $w_{i}$. This convention allows us to write statements such as $E(G) \cup E(H)$, if $G$ and $H$ have identical nodes.

Our next step is to show how we can test the subset relationship for strict episodes.

Lemma 1 Let $G, H \in \mathcal{S}$ be episodes with identical nodes. Let $s$ be a valid instance of both $G$ and $H$. Let $g$ and $h$ be the corresponding functions mapping nodes of $G$ and $H$ to indices of $s$, respectively. Then $g=h$.

Proof Let $v$ be a node. Assume that function $g$ maps $v$ to the $l$ th occurence of $l a b(v)$ in $s$. Since $s$ is an instance, then there are $l-1$ ancestors of $v$ in $G$ having the same label as $v$. Since $G$ and $H$ have identical nodes, $v$ also has $l-1$ ancestors in $H$. Since $s$ is an instance of $H, h$ must map $v$ to $l$ th occurrence of $l a b(v)$. This implies that $g=h$. 
Lemma 1 implies that given an episode $G$ and an instance $s$, there is only one valid function $f$ mapping nodes of $G$ to indices of $s$. Let us denote this mapping by $\operatorname{map}(G, s)=f$. If $G$ is a parallel episode with nodes $V=V(G)$ we write $\operatorname{map}(V, s)$.

Crucially, we can easily compute the subset relationship between two episodes.

Theorem 1 For episodes $G, H \in \mathcal{S}$ with identical nodes, $E(G) \subseteq E(H)$ if and only if $G \preceq H$.

Proof To prove the "only if" direction assume that $E(G) \subseteq E(H)$. Let $s=\left\{s_{1}, \ldots, s_{N}\right\}$ be an instance of $H$ and let $f=\operatorname{map}(H, s)$ be the corresponding mapping. Then $f$ is also a valid mapping for $G$. Thus, $G \preceq H$.

To prove the other direction, assume that $E(G) \nsubseteq E(H)$. We therefore must have an edge $e=(x, y) \in E(G)$, such that $e \notin E(H)$. We build $s$ by first visiting every parent of $y$ in $H$ in a valid order with respect to $H$, then $y$ itself, and then the rest of the nodes, also in a valid order. Let $h$ be the visiting order of $G$ while constructing $s$, that is, $h(v)=1$, if we visited $v$ first, $h(v)=2$, if we visited $v$ second. Note that $h(y)<h(x)$. Assume now that $s$ covers $G$ and let $f=\operatorname{map}(G, s)$ be the corresponding mapping. But then Lemma 1 implies that $g=h$, thus $g(y)<g(x)$, contradicting the fact that $(x, y) \in E(G)$.

Theorem 1 essentially shows that our subset relationship is in fact a graph subset relationship which allows us to design an efficient mining algorithm.

We finish this section by defining what we exactly mean when we say that two episodes are equivalent and demonstrate that the class of strict episodes contains all parallel episodes.

Definition 9 Episodes $G$ and $H$ are said to be equivalent, denoted by $G \sim H$, if each sequence that covers $G$ also covers $H$, and vice versa.

Corollary 1 (of Theorem 1) For episodes $G, H \in \mathcal{S}, G \sim H$ if and only if $E(G)=$ $E(H)$ and $G$ and $H$ have identical nodes.

Proof This follows from the fact that $G \sim H$ is equivalent to $G \preceq H$ and $H \preceq G$, and that $E(G)=E(H)$ is equivalent to $E(G) \subseteq E(H)$ and $E(H) \subseteq E(G)$.

Note that by generating only transitively closed strict episodes, we have obtained an efficient way of computing the subset relationship between two episodes. At first glance, though, it may seem that we have completely omitted certain parallel episodes from consideration - namely, all non-strict parallel episodes (i.e. those containing multiple nodes with same labels). Note, however, that for each such episode $G$, there exists a strict episode $H$, such that $G \sim H$. To build such an episode $H$, we just need to create edges that would strictly define the order among nodes with the same labels. From now on, when we talk of parallel episodes, we actually refer to their strict equivalents.

\section{Closure}

Having defined a subset relationship among episodes, we are now able to speak of an episode being more specific than another episode. However, this is only the first step towards defining the closure of an episode. We know that the closure must be more specific, but it must also be unique and well-defined. We have already seen that basing 
such a closure on the frequency fails, as there can be multiple more specific closed episodes that could be considered as closures.

In this section we will establish three closure operators, based on the instances of the episode found within the sequence. The first closure adds nodes, the second one adds edges, and the third is a combination of the first two. We also show that these operators satisfy three important properties. We will use these properties to prove the correctness of our mining algorithm. The needed properties for a closure operator $h$ are

1. Extension: $G \preceq h(G)$,

2. Idempotency: $h(G)=h(h(G))$,

3. Monotonicity: $G_{1} \preceq G_{2} \Rightarrow h\left(G_{1}\right) \preceq h\left(G_{2}\right)$.

These properties are usually shown using the Galois connection but to avoid cumbersome notation we will prove them directly.

\subsection{Node Closure}

In this section we will define a node closure and show that it satisfies the properties. Assume that we are given a sequence $s$ and a window size $\rho$.

Our first step is to define a function $f_{N}$ which maps an episode $G \in \mathcal{S}$ to a set of intervals which contain all instances of $G$,

$f_{N}(G ; s)=\left\{[\min m, \max m] \mid s_{m}\right.$ covers $\left.G, \max m-\min m<\rho, m \in M\right\}$,

where $M$ contains all strictly increasing mappings to $s$.

Our next step is to define $X_{G}$ to be the set of all symbols occurring in each interval,

$$
X_{G}=\left\{x \in \Sigma \mid x \text { occurs in } s[a, b] \text { for all }[a, b] \in f_{N}(G)\right\} .
$$

Let $W$ be the labels of the nodes of $G$. We define our first closure operator, $i c l_{N}(G)$ to be $G$ augmented with nodes having the labels $X_{G}-W$, that is, we add nodes to $G$ with labels that occur inside each window that contains $G$.

Theorem $2 i l_{N}(G)$ is an idempotentic and monotonic extension operator.

Proof The extension property follows immediately because we are only adding new nodes.

Assume now that $G \preceq H$. Let $[a, b] \in f_{N}(H)$ be an interval. Then, there is an interval $[c, d] \in f_{N}(G)$ such that $a \leq c \leq d \leq b$. This means that any symbol occurring in every interval in $f_{N}(G)$ will also occur in every interval in $f_{N}(H)$, that is, $X_{G} \subseteq X_{H}$.

Let $\alpha$ be a graph homomorphism such that $G \preceq \alpha H$. Let $x \in X_{G}$ be a symbol not occurring in $G$ and let $v$ be the new node in $i c l_{N}(G)$ with this label. If $x$ does not occur in $H$, then $x \in X_{H}$ and thus a node with a label $x$ is added into $H$. In any case, there is a node $w$ with a label $x$ in $i c l_{N}(H)$. We can extend $\alpha$ by setting $\alpha(v)=w$. By doing this for each new node we have proved monotonicity, i.e. that $i c l_{N}(G) \preceq i c l_{N}(H)$.

To prove idempotency, let us write $H=i c l_{N}(G)$. Since any new node in $H$ must occur inside the instances of $G$, we have $f_{N}(G) \subseteq f_{N}(H)$. This implies that $X_{H} \subseteq X_{G}$ and since we saw before that $X_{G} \subseteq X_{H}$, it implies that $X_{G}=X_{H}$. Since for every label in $X_{H}$ there is a node in $H$ with the same label, it holds that $i c l_{N}(H)=H$. 
Note that the node closure adds only events with unique labels. The reason for this is that if we add node $x$ to an episode containing node $y$ such that $l a b(x)=l a b(y)$, then we would have to connect $x$ and $y$. This may reduce the instances and invalidate the proof. For the same reason, we only add a maximum of one new node with a particular label. In other words, if each window containing episode $G$ also contains two occurrences of $a$, we will only add one node with label $a$ to $G$ (provided $G$ does not contain a node labelled $a$ already).

\subsection{Edge Closure}

We begin by introducing the concept of a maximal episode that is covered by a given set of sequences.

Definition 10 Given a set of nodes $V$, and a set $\mathrm{S}$ of instances of $\mathrm{V}$, interpreted as a parallel episode, we define the maximal episode covered by set $S$ as the episode $H$, where $V(H)=V$ and

$$
E(H)=\{(x, y) \in V \times V \mid f(x)<f(y), f=\operatorname{map}(V, s) \text { for all } s \in S\},
$$

where $\operatorname{map}(V, s)$ refers to the mapping defined in Lemma 1 and $V$ is interpreted as a parallel episode.

To define a closure operator we first define a function mapping an episode $G$ to all of its valid instances in a sequence $s$,

$$
f_{E}(G ; s)=\left\{s_{m} \mid s_{m} \text { covers } G, \max m-\min m<\rho, m \in M\right\},
$$

where $M$ contains all strictly increasing mappings to $s$. When the sequence is known from the context, we denote simply $f_{E}(G)$

We define $i c l_{E}(G)$ to be the maximal episode covered by $f_{E}(G)$. If $i c l_{E}(G)=G$, then we call $G$ an $e$-closed episode.

Theorem $3 \operatorname{icl}_{E}(G)$ is an idempotentic and monotonic extension operator.

Proof To prove the extension property assume an edge $\left(v_{i}, v_{j}\right) \in E(G)$. Let $V$ be the nodes in $G$. Let $w \in f_{E}(G)$ be an instance of $G$ and let $f=\operatorname{map}(V, w)$ be the corresponding mapping. Lemma 1 implies that $\operatorname{map}(V, w)=\operatorname{map}(G, w)$. Hence $f\left(v_{i}\right)<$ $f\left(v_{j}\right)$. Since this holds for every map, we have $\left(v_{i}, v_{j}\right) \in E\left(i c l_{E}(G)\right)$.

To prove the idempotency, let $H=i c l_{E}(G)$. The extension property implies that $G \preceq H$ so by definition $f_{E}(H) \subseteq f_{E}(G)$. But any instance in $f_{E}(G)$ also covers $H$. Thus, $f_{E}(H) \subseteq f_{E}(G)$ and so $f_{E}(H)=f_{E}(G)$. This implies the idempotency.

Assume now that $G \preceq H$. Let $\alpha$ be the graph homomorphism such that $G \preceq \alpha$ $H$. We will show that $i c l_{E}(G) \preceq_{\alpha} i c l_{E}(H)$. Let $(x, y) \in E\left(i c l_{E}(G)\right)$. Let $w$ be an instance of $H$ and let $f=\operatorname{map}(H, s)$ the corresponding mapping to $w$. Assume that $f(\alpha(x)) \geq f(\alpha(y))$. Let $v$ be the subsequence of $w$ containing only the indices in the range of $f \circ \alpha$. Note that $v$ is a valid instance of $G$ and $f \circ \alpha=\operatorname{map}(V(G), v)$. This contradicts the fact that $(x, y) \in E\left(i_{E l}(G)\right)$. Hence, $f(\alpha(x))<f(\alpha(y))$. This implies that $(\alpha(x), \alpha(y)) \in E\left(i_{E}(H)\right)$ which completes the proof. 
Example 5 Consider sequence $s$ given in Figure $2(\mathrm{e})$ and episode $G_{4}$ given in Figure 2(d) and assume that the window length is 5 . There are four instances of $G_{4}$ in $s$, namely $a b c d, a c d b, a c b d$ and $a b c d$. Therefore, $f_{E}\left(G_{4}\right)=\{a b c d, a c b d\}$. The serial episodes corresponding to these subsequences are $G_{2}$ and $G_{3}$ given in Figure 2. By taking the intersection of these two episodes we obtain $G_{1}=i c l_{E}\left(G_{4}\right)$ given in Figure $2(\mathrm{a})$.

\subsection{Combining Closures}

We can combine the node closure and the edge closure into one operator.

Definition 11 Given an episode $G \in \mathcal{S}$, we define $i c l_{E N}(G)=i c l_{E}\left(i c l_{N}(G)\right)$. To simplify the notation, we will refer to $i_{c l}(G)$ as $i c l(G)$, the $i$-closure of $G$. We will say that $G$ is $i$-closed if $G=i c l(G)$.

Theorem $4 \mathrm{icl}(G)$ is an idempotentic and monotonic extension operator.

Proof The extension and monotonicity properties follow directly from the fact that both $i c l_{N}(G)$ and $i c l_{E}(G)$ are monotonic extension operators.

To prove idempotency let $H=i c l(G)$ and $H^{\prime}=i c l_{N}(G)$. Since any instance of $H=i c l_{E}\left(H^{\prime}\right)$ is also an instance of $H^{\prime}$, and, per definition, vice versa, we see that $f_{N}(H)=f_{N}\left(H^{\prime}\right)$, and consequently $i c l_{N}(H)=H$, and $i c l(H)=i c l_{E}\left(i c l_{N}(H)\right)=$ $i c l_{E}(H)=H$.

The advantage of mining $i$-closed episodes instead of $e$-closed is prominent if the sequence contains a long sequential pattern. More specifically, assume that the input sequence contains a frequent subsequence of $N$ symbols $p_{1}, \ldots, p_{N}$, and no other permutation of this pattern occurs in the sequence. The number of $e$-closed subpatterns of this subsequence is $2^{N}-1$, namely, all non-empty serial subepisodes. However, the number of its $i$-closed subpatterns is $N(N+1) / 2$, namely, serial episodes of form $p_{i} \rightarrow \cdots \rightarrow p_{j}$ for $1 \leq i \leq j \leq N$.

\subsection{Computing Closures}

During the mining process, we need to compute the closure of an episode. The definition of closures use $f_{N}(G)$ and $f_{E}(G)$ which are based on instances of $G$ in $s$. However, there can be an exponential number of such instances in $s$.

In the following discussion we will often use the following notations. Given an episode $G$, we write $G+v$ to mean the episode $G$ augmented with an additional node $v$. Similarly, we will use the notations $G+e$ and $G+V$, where $e$ is an edge and $V$ is a set of nodes. We also use $G-v, G-V$, and $G-e$ to mean episodes where either nodes or edges are removed.

To avoid the problem of an exponential number of instances we make two observations: Firstly, a node with a new label $l$ is added into the closure if and only if it occurs in every minimal window of $G$. Secondly, an edge $(x, y)$ is added into the closure if and only if there is no minimal window for $G+(y, x)$. Thus to compute the closure we need an algorithm that finds all minimal windows of $G$ in $s$. Note that, unlike with instances of $G$, there can be only $|s|$ minimal windows.

Using strict episodes allows us to discover minimal windows in an efficient greedy fashion. 
Lemma 2 Given an episode $G \in \mathcal{S}$ and a sequence $s=s_{1} \cdots s_{L}$, let $k$ be the smallest index such that $s_{k}=l a b(v)$, where $v$ is a source node in $G$. Then $s$ covers $G$ if and only if $s[k+1, L]$ covers $G-v$.

Proof Let $f$ be a mapping from $V(G)$ to $s$. If $k$ is not already used by $f$, then we can remap a source node $v$ to $k$. As $k$ is the smallest entry used by $f$, the remaining map is a valid mapping for $G-v$ in $s[k+1, L]$.

Lemma 2 says that to test whether a sequence $s$ covers $G$, it is sufficient to greedily find entries from $s$ corresponding to the sources of $G$, removing those sources as we move along. Let us denote such a mapping by $g(G ; s)$. Note that if the episode is not strict, then we can have two source nodes with the same label, in which case it might be that Lemma 2 holds for one of the sources but not for the other. Since we cannot know in advance which node to choose, this ambiguity would make the greedy approach less efficient.

Lemma 3 Let $G \in \mathcal{S}$ be an episode and let $s$ be a sequence covering $G$. Let $m=g(G ; s)$ and let $[a, b]$ be the first minimal window of $G$ in $s$. Then $\max m=b$.

Proof Since $s[1, b]$ covers $G$, there is a mapping $m^{\prime}=g(G ; s[1, b])$. Since $[a, b]$ is the first minimal window, we must have $\max m^{\prime}=b$. Since $m$ and $m^{\prime}$ are constructed in a greedy fashion, we must have $m=m^{\prime}$.

Lemma 3 states that if we evaluate $m=g(G ; s[k, L])$ for each $k=1, \ldots, K$, store the intervals $W=[\min m, \max m]$, and for each pair of windows $\left[a_{1}, b\right],\left[a_{2}, b\right] \in W$ remove $\left[a_{2}, b\right]$, then $W$ will contain the minimal windows. Evaluating $g(G ; s)$ can be done in polynomial time, so this approach takes polynomial time. The algorithm for discovering minimal windows is given in Algorithm 2. To fully describe the algorithm we need the following definition.

Definition 12 An edge $(v, w)$ in an episode $G \in \mathcal{S}$ is called a skeleton edge if there is no node $u$ such that $(v, u, w)$ is a path in $G$. If $v$ and $w$ have different labels, we call the edge $(v, w)$ a proper skeleton edge.

\section{Theorem 5 Algorithm FindWindows discovers all minimal windows.}

Proof We will prove the correctness of the algorithm in several steps. First, we will show that the loop 8-16 guarantees that $f$ is truly a valid mapping. To see this, note that the loop upholds the following invariants: For every $v \notin Q$, we have $f(v)>b(v)$ and for each skeleton edge $(v, w)$, we have $b(w) \neq-\infty$. Also, for every non-source node $w, b(w)=\max \{f(v) \mid(v, w)$ is a skeleton edge $\}$ or $b(w)=-\infty$.

Thus when we leave the loop, that is, $Q=\emptyset$, then $f$ is a valid mapping for $G$. Moreover, since we select the smallest $f(v)$ during Line 11, we can show using recursion that $f=g(G ; s[k+1, L])$, where $k=\min b(v)$, where the min ranges over all source nodes. Once $f$ is discovered, the next new mapping should be contained in $s[\min f+$ $1, L]$. This is exactly what is done on Line 22 by making $b(v)$ equal to $f(v)$.

The advantage of this approach is that we can now optimize the search on Line 11 by starting the search from the previous index $f(v)$ instead of starting from the start of the sequence. The following lemma, implied directly by the greedy procedure, allows this. 


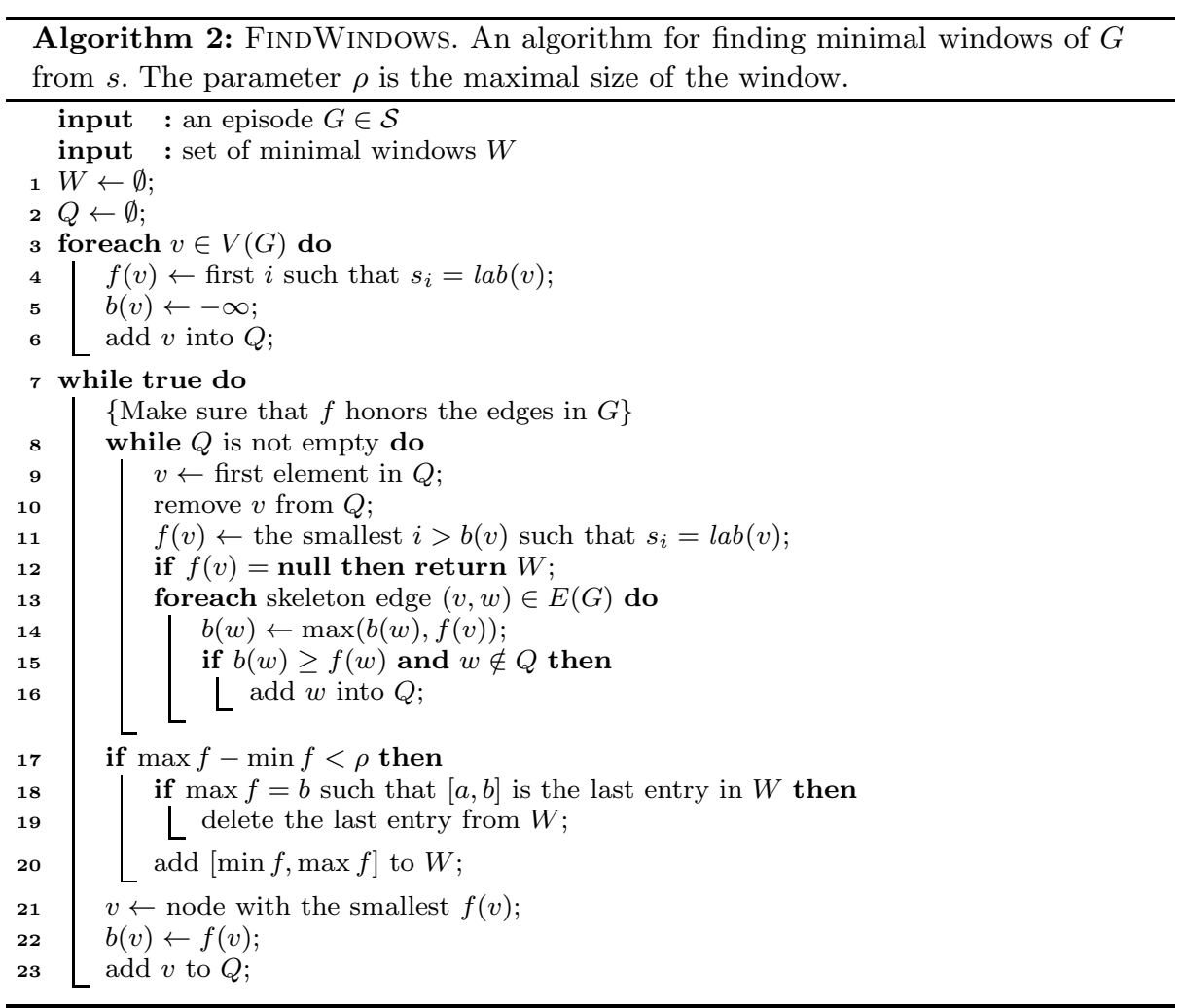

Lemma 4 Let $G \in \mathcal{S}$ be an episode and let $s$ be a sequence covering $G$. Let $k$ be an index and assume that $s[k, L]$ covers $G$. Set $m_{1}=g(G ; s)$ and $m_{2}=g(G ; s[k+1, L])$. Then $m_{2}(v) \geq m_{1}(v)$ for every $v \in V(G)$.

Let us now analyze the complexity of FindWindows. Assume that the input episode $G$ has $N$ nodes and the input sequence $s$ has $L$ symbols. Let $K$ be the maximal number of nodes in $G$ sharing the same label. Let $D$ be the maximal number of outgoing skeleton edges in a single node. Note that each event in $s$ is visited during Line $11 K$ times, at maximum. Each visit may require $D$ updates of $b(w)$, at maximum. The only remaining non-trivial step is finding the minimal source (Line 21), which can be done with a heap in $O(\log N)$ time. This brings the total evaluation time to $O((D+1) K L+L \log N)$. Note that for parallel episodes $D=0$ and for serial episodes $D=1$, and for such episodes we can further optimize the algorithm such that each event is visited only twice, thus making the running time to be in $O(L \log N)$ for parallel episodes and in $O(L)$ for serial episodes. Since there can be only $L$ minimal windows, the additional space complexity for the algorithm is in $O(L)$.

Given the set of minimal windows, we can now compute the node closure by testing which nodes occur in all minimal windows. This can be done in $O(L)$ time. To compute the edge closure we need to perform $N^{2}$ calls of FindWIndows. This can be greatly optimized by sieving multiple edges simultaneously based on valid mappings $f$ discovered during FindWindows. In addition, we can compute both frequency measures from minimal windows in $O(L)$ time. 
We will now turn to discovering $f$-closed episodes. Note that, unlike the $i$-closure, we do not define an $f$-closure of an episode at all. As shown in Section 1, such an $f$-closure would not necessarily be unique. However, the set of $i$-closed episodes will contain all $f$-closed episodes.

Theorem 6 An f-closed episode is always $i$-closed.

Proof Let $G \in \mathcal{S}$ be an episode and define $H=i c l(G)$. Let $W$ be a set of all minimal windows of $s$ that cover $G$ and let $V$ be the set of all minimal windows that cover $H$. Let $w=[a, b] \in W$ be a minimal window of $G$ and let $f$ be a valid mapping from $G$ to $s[a, b]$. Any new node added in $H$ must occur in $s[a, b]$ and $f$ can be extended to $H$ such that it honours all edges in $H$. Hence $s[a, b]$ covers $H$. It is also a minimal window for $H$ because otherwise it would violate the minimality for $G$. Hence $w \in V$. Now assume that $v=\left[a^{\prime}, b^{\prime}\right] \in V$. Obviously, $s\left[a^{\prime}, b^{\prime}\right]$ covers $G$, so there must be a minimal window $w=[a, b] \in W$. Using the first argument we see that $w \in V$. Since $V$ contains only minimal windows we conclude that $v=w$. Hence $W=V$. A sequence covers an episode if and only if the sequence contains a minimal window of the episode. Thus, by definition, $V=W$ implies that $f r_{f}(G)=f r_{f}(H)$ and $f r_{d}(G)=f r_{d}(H)$.

Assume now that $G$ is not $i$-closed, then $G \neq H$, and since $\operatorname{fr}(G)=\operatorname{fr}(H), G$ is not $f$-closed either. This completes the proof.

A naïve approach to extract $f$-closed episodes would be to compare each pair of instance-closed episodes $G$ and $H$ and if $\operatorname{fr}(G)=\operatorname{fr}(H)$ and $G \prec H$, remove $G$ from the output. This approach can be considerably sped up by realising that we need only to test episodes with identical nodes and episodes of form $G-V$, where $V$ is a subset of $V(G)$. The pseudo-code is given in Algorithm 3. The algorithm can be further sped up by exploiting the subset relationship between the episodes. Our experiments demonstrate that this comparison is feasible in practice.

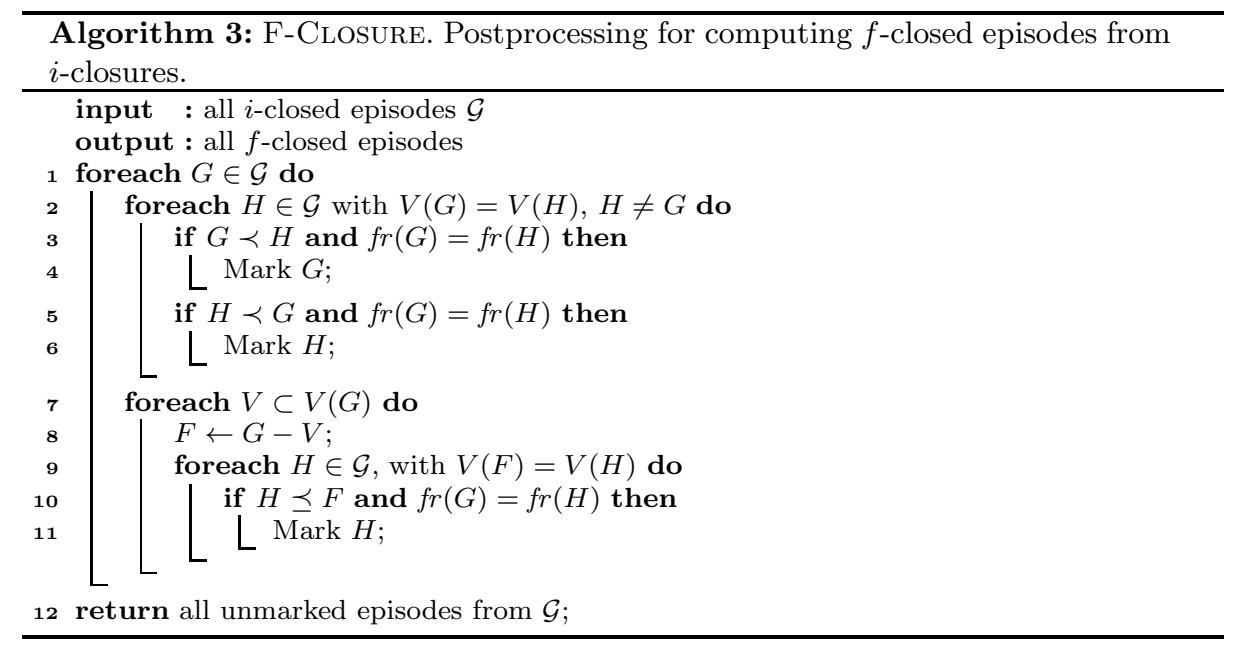




\section{Generating Transitively Closed Candidate Episodes}

In this section we define an algorithm, GENERATECANDIDATE, which generates the candidate episodes from the episodes discovered previously. The difficulty of the algorithm is that we need to make sure that the candidates are transitively closed.

Let $G \in \mathcal{S}$ be an episode. It is easy to see that if we remove a proper skeleton edge $e$ from $G$, then the resulting episode $G-e$ will be in $\mathcal{S}$, that is, transitively closed and strict. We can reverse this property in order to generate candidates: Let $G \in \mathcal{S}$ be a previously discovered episode, add an edge $e$ and verify that the new episode is transitively closed. However, we can improve on this naïve approach with the following theorem describing the sufficient and necessary condition for an episode to be transitively closed.

Theorem 7 Let $G \in \mathcal{S}$ be an episode and let $e=(x, y)$ be an edge not in $E(G)$. Let $H=G+e$. Assume that $H$ is a $D A G$. Then $H \in \mathcal{S}$ if and only if there is an edge in $G$ from $x$ to every child of $y$ and from every parent of $x$ to $y$.

Proof The 'only if' part follows directly from the definition of transitive closure. To prove the 'if' part, we will use induction. Let $u$ be an ancestor node of $v$ in $H$. Then there is a path from $u$ to $v$ in $H$. If the path does not use edge $e$, then, since $G$ is transitively closed, $(u, v) \in E(G)$ and hence $(u, v) \in E(H)$. Assume now that the path uses $e$. If $v=y$, then $u$ must be a parent of $y$ in $G$, since $G$ is transitively closed, so the condition implies that $(u, v) \in E(G) \subset E(H)$. Assume that $v$ is a proper descendant of $y$ in $H$. To prove the first step in the induction, assume that $u=x$, then again $(u, v) \in E(G)$. To prove the induction step, let $w$ be the next node along the path from $u$ to $v$ in $H$. Assume inductively that $(w, v) \in E(G)$. Then the path $(u, w, v)$ occurs in $G$, so $(u, v) \in E(G)$, which completes the proof.

We now show when we can join two episodes to obtain a candidate episode. Since our nodes are ordered, we can also order the edges using a lexicographical order. Given an episode $G$ we define last $(G)$ to be the last proper skeleton edge in $G$. The next theorem shows the necessary conditions for the existence of the candidate episode.

Theorem 8 Let $H \in \mathcal{S}$ be an episode with $N+1$ edges. Then either there are two episodes, $G_{1}, G_{2} \in \mathcal{S}$, with identical nodes to $H$, such that

- $G_{1}$ and $G_{2}$ share $N-1$ edges,

$-e_{1}=\operatorname{last}\left(G_{1}\right) \notin E\left(G_{2}\right)$,

- $e_{2}>e_{1}$ and $H=G_{1}+e_{2}$, where $e_{2}$ is the unique edge in $E\left(G_{2}\right)$ and not in $E\left(G_{1}\right)^{3}$

or last $(G)$ is no longer a skeleton edge in $H$, where $G \in \mathcal{S}, G=H-\operatorname{last}(H)$.

We will refer to the two cases as Case A and Case B, respectively.

Proof Let $e_{2}=\operatorname{last}(H)$ and define $G_{1}=H-e_{2}$. If $e_{1}$ is not a skeleton edge in $H$, then by setting $G=G_{1}$, the theorem holds. Assume that $e_{1}$ is a skeleton edge in $H$. Define $G_{2}=H-e_{1}$. Note that $G_{1}$ and $G_{2}$ share $N-1$ edges. Also note that $\operatorname{last}\left(G_{1}\right)=e_{1} \notin E\left(G_{2}\right),\left\{e_{2}\right\}=E\left(G_{2}\right)-E\left(G_{1}\right)$, and $H=G_{1}+e_{2}$. Since $e_{2}=\operatorname{last}(H)$ it must be that $e_{2}>e_{1}$.

\footnotetext{
${ }^{3}$ In [17] we incorrectly stated that $e_{2}=\operatorname{last}\left(G_{2}\right)$. Generally, this is not the case.
} 
Theorem 8 gives us means to generate episodes. Let us first consider Case A in Theorem 8. To generate $H$ we simply find all pairs of episodes $G_{1}$ and $G_{2}$ such that the conditions of Case A in Theorem 8 hold. When combining $G_{1}$ and $G_{2}$ we need to test whether the resulting episode is transitively closed.

Theorem 9 Let $G_{1}, G_{2} \in \mathcal{S}$ be two episodes with identical nodes and $N$ edges. Assume that $G_{1}$ and $G_{2}$ share $N-1$ mutual edges. Let $e_{1}=\left(x_{1}, y_{1}\right) \in E\left(G_{1}\right)-E\left(G_{2}\right)$ be the unique edge of $G_{1}$ and let $e_{2}=\left(x_{2}, y_{2}\right) \in E\left(G_{2}\right)-E\left(G_{1}\right)$ be the unique edge of $G_{2}$. Let $H=G_{1}+e_{2}$. Assume that $H$ has no cycles. Then $H \in \mathcal{S}$ if and only if one of the following conditions is true:

1. $x_{1} \neq y_{2}$ and $x_{2} \neq y_{1}$.

2. $x_{1} \neq y_{2}, x_{2}=y_{1}$, and $\left(x_{1}, y_{2}\right)$ is an edge in $G_{1}$.

3. $x_{1}=y_{2}, x_{2} \neq y_{1}$, and $\left(x_{2}, y_{1}\right)$ is an edge in $G_{1}$.

Moreover, if $H \in \mathcal{S}$, then $e_{1}$ and $e_{2}$ are both skeleton edges in $H$.

Proof We will first show that $e_{1}$ is a skeleton edge in $H$ if $H \in \mathcal{S}$. If it is not, then there is a path from $x_{1}$ to $y_{1}$ in $H$ not using $e_{1}$. The edges along this path also occur in $G_{2}$, thus forcing $e_{1}$ to be an edge in $G_{2}$, which is a contradiction. A similar argument holds for $e_{2}$.

The "only if" part is trivial so we only prove the "if" part using Theorem 7 .

Let $v$ be a child of $y_{2}$ in $G_{1}$ and $f=\left(y_{2}, v\right)$ an edge in $G_{1}$.

If the first or second condition holds, then $x_{1} \neq y_{2}$, and consequently $f \neq e_{1}$, so $f \in G_{2}$. The path $\left(x_{2}, y_{2}, v\right)$ connects $x_{2}$ and $v$ in $G_{2}$ so there must be an edge $h=\left(x_{2}, v\right)$ in $G_{2}$. Since $h \neq e_{2}, h$ must also occur in $G_{1}$. If the third condition holds, it may be the case that $f=e_{1}$ (if not, then we can use the previous argument). But in such a case $v=y_{1}$ and edge $h=\left(x_{2}, y_{1}\right)$ occurs in $G_{1}$.

If now $u$ is a parent of $x_{2}$ in $G_{1}$, we can make a similar argument that $u$ and $y_{2}$ are connected, so Theorem 7 now implies that $H$ is transitively closed.

Theorem 9 allows us to handle Case A of Theorem 8. To handle Case B, we simply take an episode $G$ and try to find all edges $e_{2}$ such that last $\left(G+e_{2}\right)=e_{2}$ and $\operatorname{last}(G)$ is no longer a skeleton edge in $G+e_{2}$. The conditions for this are given in the next theorem.

Theorem 10 Let $G \in \mathcal{S}$ be an episode, let $e_{1}=\left(x_{1}, y_{1}\right)$ be a skeleton edge of $G$, and let $e_{2}=\left(x_{2}, y_{2}\right)$ be an edge not occurring in $G$ and define $H=G+e_{2}$. Assume $H \in \mathcal{S}$ and that $e_{1}$ is not a skeleton edge in $H$. Then either $y_{2}=y_{1}$ and $\left(x_{1}, x_{2}\right)$ is a skeleton edge in $G$ or $x_{1}=x_{2}$ and $\left(y_{2}, y_{1}\right)$ is a skeleton edge in $G$.

Proof Assume that $e_{1}$ is no longer a skeleton edge in $H$, then there is a path of skeleton edges going from $x_{1}$ to $y_{1}$ in $H$ not using $e_{1}$. The path must use $e_{2}$, otherwise we have a contradiction. The theorem will follow if we can show that the path must have exactly two edges. Assume otherwise. Assume, for simplicity, that edge $e_{2}$ does not occur first in the path and let $z$ be the node before $x_{2}$ in the path. Then we can build a new path by replacing edges $\left(z, x_{2}\right)$ and $e_{2}$ with $\left(z, y_{2}\right)$. This path does not use $e_{2}$, hence it occurs in $G$. If $z \neq x_{1}$ or $y_{1} \neq y_{2}$, then the path makes $e_{1}$ a non-skeleton edge in $G$, which is a contradiction. If $e_{2}$ is the first edge in the path, we can select the next node after $y_{2}$ and repeat the argument. 
Example 6 Consider the episodes given in Figure 5. Episodes $G_{1}$ and $G_{2}$ satisfy the second condition in Theorem 9, hence the resulting episode $H_{1}$, is transitively closed. On the other hand, combining $G_{1}$ and $G_{3}$ leads to $H_{1}^{\prime}$, an episode that is not transitively closed since edge $(a, d)$ is missing. Finally, $G_{4}$ and $(c, a)$ satisfy Theorem 10 and generate $\mathrm{H}_{2}$.

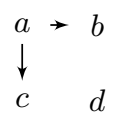

(a) $G_{1}$

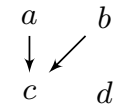

(b) $G_{2}$

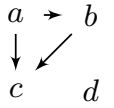

(c) $H_{1}$

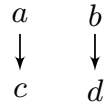

(d) $G_{3}$

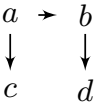

(e) $H_{1}^{\prime}$

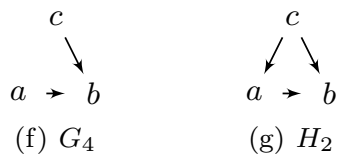

Fig. 5 Toy episodes for Example 6.

We can now combine the preceding theorems into the GenERATECANDIDATE algorithm given in Algorithm 4. We will first generate candidates by combining episodes from the previous rounds using Theorem 9. Secondly, we use Theorem 10 and for each episode from the previous rounds we add edges such that the last proper skeleton edge is no longer a skeleton edge in the candidate.

Note that Algorithm 4 will not generate duplicates. To see this, first note that if $H$ is generated using Case A, then the two episodes $G_{1}$ and $G_{2}$ generating $H$ are unique. That is, it must be that $G_{1}=H-\operatorname{last}(H)$ and $G_{2}=H-\operatorname{last}\left(G_{1}\right)$. In other words, $G_{1}$ and $G_{2}$ will produce a unique $H$. In Case B, the episode $G_{1}$ is also unique, namely, we must have $G_{1}=H-\operatorname{last}(H)$. Thus, each $G_{1}$ will produce a unique $H$. Finally, note that last $\left(G_{1}\right)$, according to Theorem 9 is always a skeleton edge in $H$ in Case A and we demand that last $\left(G_{1}\right)$ is not a skeleton edge in Case B. Hence, Case A and Case B will never generate the same episode.

\section{Algorithm for Discovering Closed Episodes}

In this section we will complete our mining algorithm for discovering closed episodes. First, we present an algorithm for testing the episodes and a more detailed version of Algorithm 1. Then we present an algorithm for adding the needed intermediate episodes and prove the correctness of the algorithm.

\subsection{Detailed version of the algorithm}

We begin by describing the test subroutine that is done for each candidate episode. Following the level-wise discovery, before computing the frequency of the episode, we need to test that all its subepisodes are discovered. It turns out that using transitively closed episodes will guarantee the strongest conditions for an episode to pass to the frequency computation stage. 


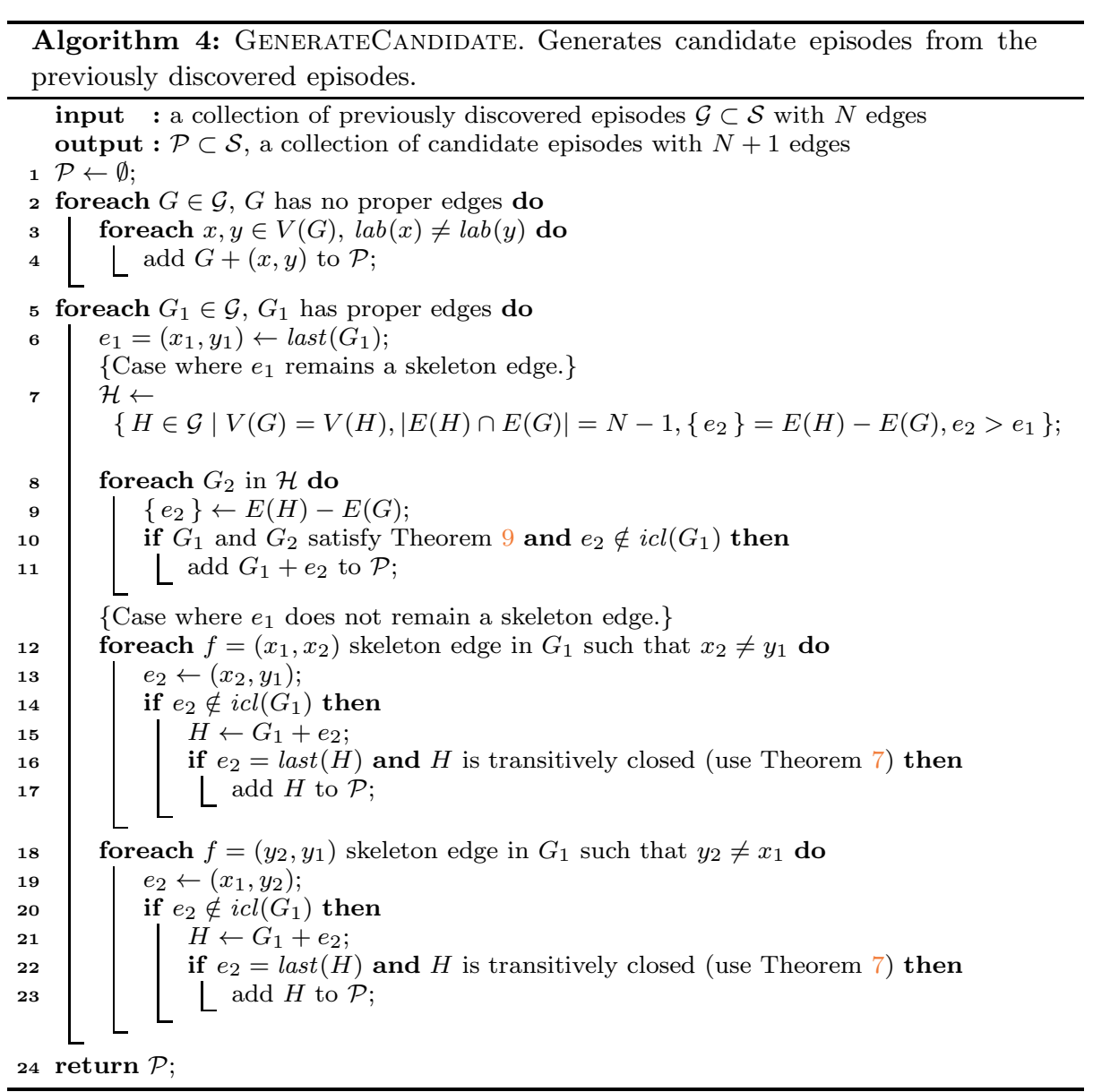

Corollary 2 (of Theorem 1) Let $G \in \mathcal{S}$ be an episode. Let e be a proper skeleton edge of $G$. If $H$ is an episode obtained by removing e from $G$, then there exists no episode $H_{1} \in \mathcal{S}$, such that $H \prec H_{1} \prec G$.

If $e$ is a proper skeleton edge of an episode $G \in \mathcal{S}$, then $G-e \in \mathcal{S}$. Thus, for $G$ to be frequent, $G-e$ had to be discovered previously. This is the first test in TESTCANDIDATE (given in Algorithm 5). In addition, following the level-wise approach for mining closed patterns [14], we test that $G$ is not a subepisode of $i c l(G-e)$, and if it is, then we can discard $G$.

The second test involves testing whether $G-v$, where $v$ is a node in $G$, has also been discovered. Note that $G-v$ has fewer nodes than $G$ so, if $G$ is frequent, we must have discovered $G-v$. Not all nodes need to be tested. If a node $v$ has an adjacent proper skeleton edge, say $e$, then the episode $G-e$ has a frequency lower than or equal to that of $G-v$. Since we have already tested $G-e$ we do not need to test $G-v$. Consequently, we need to test only those nodes that have no proper skeleton edges. This leads us to the second test in TESTCANDIDATE. Note that these nodes will either have no edges, or will have edges to the nodes having the same label. If both tests are passed we test the candidate episode for frequency. 
Example 7 Consider the episodes given in Figure 6. When testing the candidate episode $H$, TestCandidate will test for the existence of three episodes. Episodes $G_{1}$ and $G_{2}$ are tested when either edge is removed and $G_{3}$ is tested when node labelled $d$ is removed.

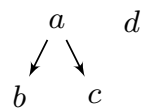

(a) $H$

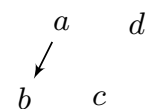

(b) $G_{1}$

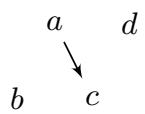

(c) $G_{2}$

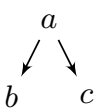

(d) $G_{3}$

Fig. 6 Toy episodes for Example 7.

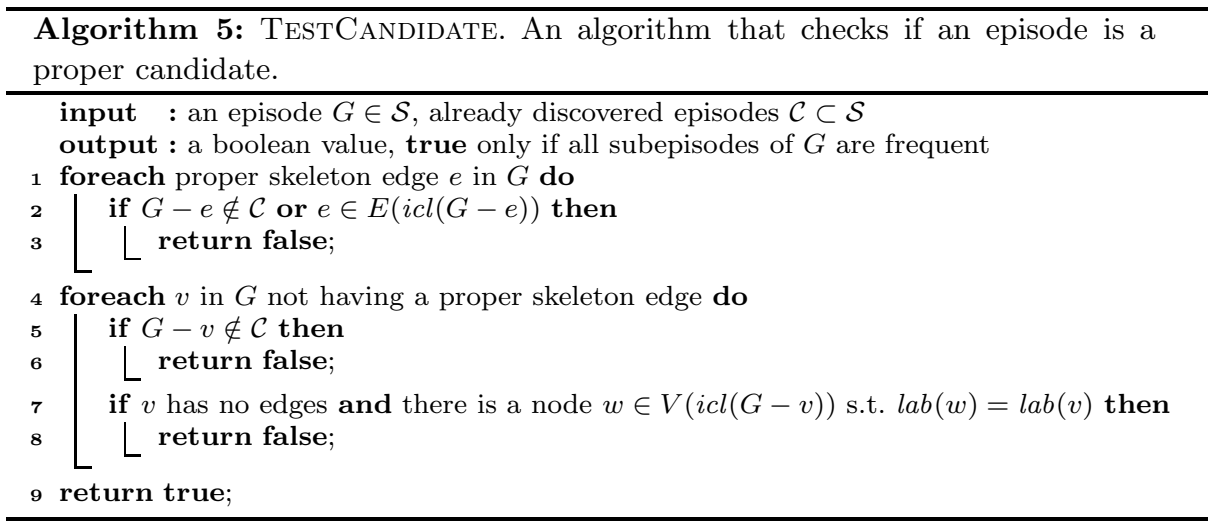

Finally we present a more detailed version of Algorithm 1, given in Algorithm 6. The only missing part of the algorithm is the ADDINTERMEDIATE subroutine which we will give in the next section along with the proof of correctness.

\subsection{Proof of Correctness}

In the original framework for mining itemsets, the algorithm discovers itemset generators, the smallest itemsets that produce the same closure. It can be shown that generators form a downward closed collection, so they can be discovered efficiently. This, however, is not true for episodes, as the following example demonstrates.

Example 8 Consider the episodes given in Figure 7 and a sequence $s=a c b x x a b c x x b a c$. Assume that the window size is $\rho=3$ and the frequency threshold is $\sigma=1$. The frequency of $H$ is 1 and there is no subepisode that has the same frequency. Hence to discover this episode all of its maximal subepisodes need to be discovered. One of these subepisodes is $i c l(G)$. However, $i c l(G)$ is not added into $\mathcal{E}$ since $(a, c) \in$ $i c l(i c l(G)-(a, c))$ and TestCandidate returns false. 


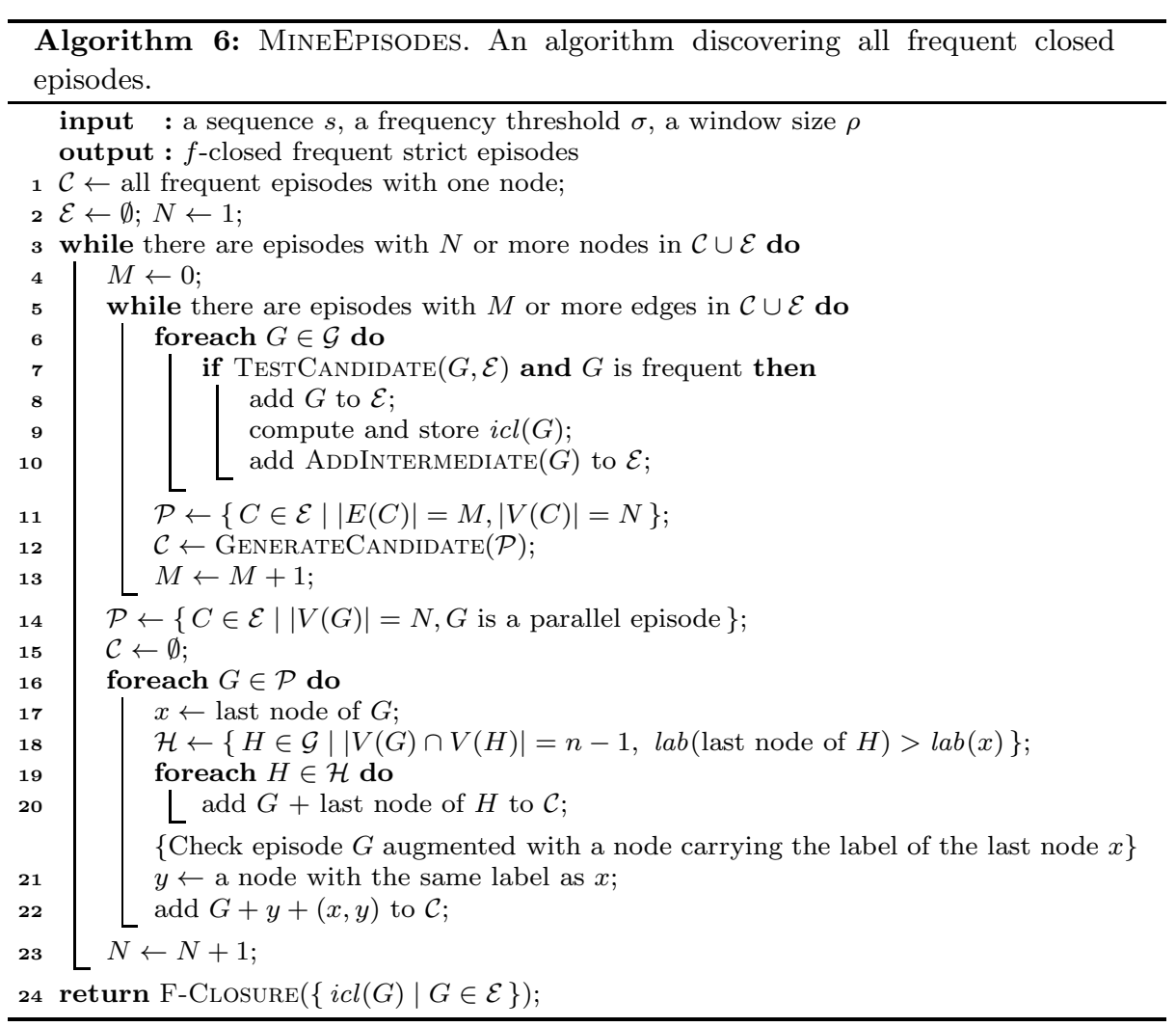

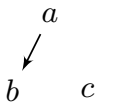

(a) $G$

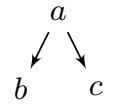

(b) $\operatorname{icl}(G)$

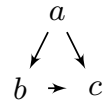

(c) $H$

Fig. 7 Toy episodes for Example 8.

The core of the problem is that sometimes adding some particular edge is not allowed until you have added another edge. For example, we cannot add $(b, c)$ to $G$ (given in Figure $7(\mathrm{a})$ ) until we have added $(a, c)$.

We solve this problem by adding some additional episodes between the discovered episode $G$ and its closure $i c l(G)$. Roughly speaking, given an episode $G \in \mathcal{S}$ we need to add the episodes containing edges/nodes from $i c l(G)$ such that these edges/nodes can be "hidden" by a single edge or node.

The details are given in the AddIntermediate algorithm given in Algorithm 7, and the justification for adding these episodes is given in the proof of Theorem 11 .

We will now establish the correctness of the algorithm and justify Algorithm 7.

Definition 13 Let $G \in \mathcal{S}$ be an episode. We call a proper skeleton edge $e$ in $G$ derivable if $G \preceq i c l(G-e)$. We call a solitary node $v$ derivable if $G \preceq i c l(G-v)$.

We will need the following straightforward lemmae to prove our main result. 


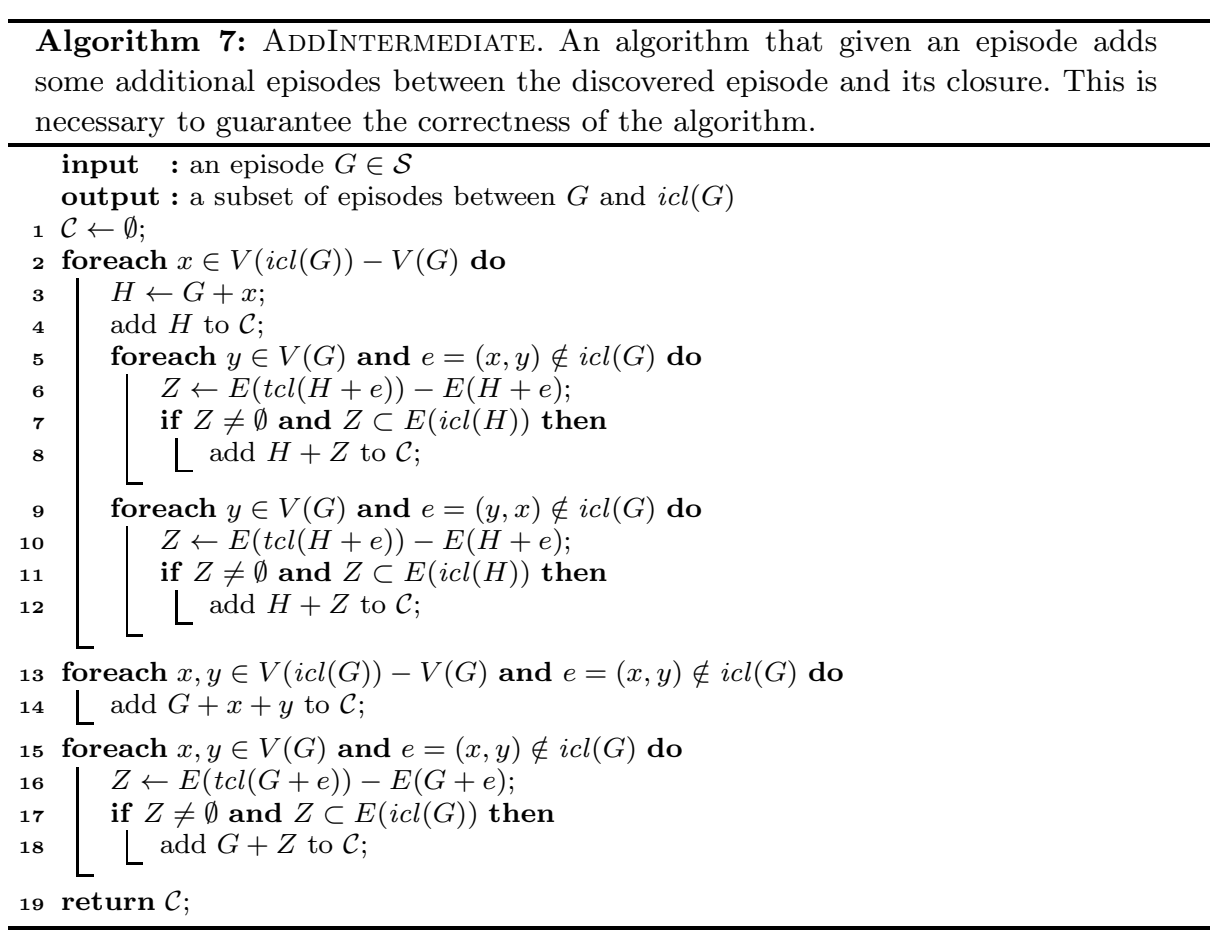

Lemma 5 Let $G \in \mathcal{S}$ be an episode. Let e be a non-derivable skeleton edge in $G$. Then $e$ is a non-derivable skeleton edge in $G-f$, where $f \neq e$ is a proper skeleton edge in $G$. Similarly, e is a non-derivable skeleton edge in $G-v$, where $v$ is a solitary node.

Proof Assume that $e$ is derivable in $G-f$, then $G-f \preceq i c l(G-f-e) \preceq i c l(G-e)$. On the other hand, $G-e \preceq i c l(G-e)$. Since the closure operator adds nodes only with unique labels, the graph homomorpisms from $G-e$ to $i c l(G-e)$ and from $G-f$ to $i c l(G-e)$ must be equal. This implies that $G \preceq i c l(G-e)$ which is a contradiction. A similar argument holds for $G-v$.

Lemma 6 Let $G \in \mathcal{S}$ be an episode. Let $w$ be a non-derivable solitary node in $G$. Then $w$ is a non-derivable node in $G-v$, where $v$ is a solitary node. Similarly, $w$ is a non-derivable node in $G-e$, where $e$ is a proper skeleton edge in $G$.

Proof Similar to the proof of Lemma 5.

Lemma 7 Let $G \in \mathcal{S}$ be an episode with a derivable edge e. Then $i c l(G)=i c l(G-e)$. Let $G \in \mathcal{S}$ be an episode with a derivable node $v$. Then $i c l(G)=i c l(G-v)$.

Proof The monotonicity and idempotency properties imply $i c l(G) \preceq \operatorname{icl}(i \operatorname{cl}(G-e))=$ $i c l(G-e)$. Since $i c l(G-e) \preceq i c l(G)$, we have $i c l(G)=i c l(G-e)$.

To prove the second case, note that $i c l(G) \preceq \operatorname{icl}(i c l(G-v))=i c l(G-v)$ which immediately implies that $i c l(G)=i c l(G-v)$.

Theorem 11 Assume a frequent episode $G \in \mathcal{S}$ with no derivable edges or derivable nodes. Then $G$ is discovered. 
Proof We will prove the theorem by induction. Obviously, the theorem holds for episodes with a single node. Assume now that the theorem is true for all subepisodes of $G$. Episode $G$ will be discovered if it passes the tests in TestCAndidate. To pass these tests, all episodes of form $G-e$, where $e$ is a proper skeleton edge need to be discovered. Assume that all these subepisodes are discovered but we have $e \in E(i c l(G-e))$. This means, by definition, that $e$ is a derivable edge in $G$ which is a contradiction. The same argument holds for episodes of form $G-v$, where $v$ is a node.

Now assume that one of the subepisodes, say, $H$ is not discovered. The induction assumption now implies that $H$ has either derivable nodes or edges.

Lemma 8 There is a subepisode $F \in \mathcal{S}, F \prec H$ with no derivable edges and nodes such that $H \preceq i c l(F)$. Any non-derivable skeleton edge in $H$ will remain in $F$. Any non-derivable solitary node in $H$ will remain in $F$.

Proof Build a chain of episodes $H=H_{1}, H_{2}, \ldots, H_{N}=F$ such that $H_{i+1}$ is obtained from $H_{i}$ by removing either a derivable node or a derivable edge and $F$ has no derivable edges or nodes. Note that this sequence always exists but may not necessarily be unique. Lemma 7 implies that we have $H_{i} \preceq i c l\left(H_{i}\right)=i c l\left(H_{i+1}\right)$. Idempotency and monotonicity imply that $H \preceq \operatorname{icl}(F)$. Lemma 5 implies that if $e$ is a non-derivable skeleton edge in $H$, then $e$ is also a non-derivable skeleton edge in each $H_{i}$. Similarly, Lemma 6 implies that any non-derivable solitary node will remain in each $H_{i}$.

By the induction assumption $F$ is discovered. We claim that $H$ will be discovered by Addintermediate $(F)$. Let us denote $Z=E(H)-E(F)$ and $W=V(H)-V(F)$.

The next three lemmae describe different properties of $Z$ and $W$.

Lemma 9 Assume that $H=G-v$. Then $W=\{w\}$ such that $l a b(v)=l a b(w)$ and $Z=\emptyset$.

Proof Lemma 5 implies that all skeleton edges in $H$ are non-derivable. Lemma 8 implies that $Z=\emptyset$. Removing $v$ can turn only one node, say $w$, into a solitary node. This happens when $l a b(v)=l a b(w)$ and there are no other edges adjacent to $w$.

Lemma 10 Assume that $H=G-(x, y)$. Then $W \subseteq\{x, y\}$. If $W=\{x, y\}$, then $Z=\emptyset$.

Proof Let $z$ be a node in $G$ (and in $H$ ) such that $z \notin\{x, y\}$. If $z$ is a solitary node in $G$, it also a solitary node in $H$. Lemma 8 now implies that $z \notin W$. If $z$ has an adjacent skeleton edge in $G$, say $f$, then $f \neq(x, y)$. Lemma 5 implies that $f$ is also a non-derivable skeleton edge in $H$. Lemma 8 now implies that $z \notin W$.

If $W=\{x, y\}$, then $x$ (and $y$ ) cannot have any adjacent non-derivable skeleton edges in $H$. Hence there are no edges, except for $(x, y)$, adjacent to $x$ or $y$ in $G$. Lemma 5 implies that all skeleton edges in $H$ are non-derivable. Lemma 8 implies that $Z=\emptyset$.

Lemma 11 Assume that $H=G-e$. Then $Z=E(t c l(F+W+e))-E(F+W+e) \subset$ $E(i c l(F+W))$.

Note that $F+W+e$ is not necessarily transitively closed.

Proof Write $F^{\prime}=F+W$. First note that $Z \subset E(H) \subseteq E\left(i c l\left(F^{\prime}\right)\right)$. Lemmae 5 and 8 imply that all skeleton edges of $G$, except for $e$ are in $E(F)=E\left(F^{\prime}\right)$. Hence, we must have $E\left(t c l\left(F^{\prime}+e\right)\right)=E(G)$.

Also note that $Z \cup E\left(F^{\prime}+e\right)=E(H)+e=E(G)$. Since $Z \cap E\left(F^{\prime}+e\right)=\emptyset$, we have $Z=E(G)-E\left(F^{\prime}+e\right)=E\left(t c l\left(F^{\prime}+e\right)\right)-E\left(F^{\prime}+e\right)$. 
If $H=G-v$, then Lemma 9 implies that $H=F+w$ and we discover $H$ on Line 4 during Addintermediate $(F)$. Assume that $H=G-e$. Write $(x, y)=e$. Lemma 10 now implies that $W \subseteq\{x, y\}$ and Lemma 11 implies that $Z=E(\operatorname{tcl}(F+W+e))-$ $E(F+W+e)$. We will show that there are 4 different possible cases:

1. $W=\{x, y\}$. Lemma 10 implies that $Z=\emptyset$. This implies that $H=F+x+y$ and we discover $H$ on Line 14 during $\operatorname{Addintermediate}(F)$.

2. $W=\{w\}$, where $w$ is either $x$ or $y$ and $Z=\emptyset$. This implies that $H=F+w$ and we discover $H$ on Line 4 during Addintermediate $(F)$.

3. $W=\emptyset$ and $Z \neq \emptyset$. This implies that $H=F+Z$ and we discover $H$ on Line 18 during Addintermediate $(F)$.

4. $W=\{w\}$, where $w$ is either $x$ or $y$ and $Z \neq \emptyset$. This implies that $H=F+w+Z$ and we discover $H$ either on Line 8 or on Line 12 during $\operatorname{Addintermediate}(F)$.

This compeletes the proof of the theorem.

Theorem 12 Every frequent $i$-closed episode will be outputted.

Proof TestEpisode will output $i c l(G)$ for each discovered episode $G$. Hence, we need to show that for each $i$-closed episode $H$ there is a discovered episode $G$ such that $H=i c l(G)$.

We will prove the theorem by induction. Let $H$ and $G$ be episodes such that $H=$ $i c l(G)$ and $H$ is an $i$-closed frequent episode. If $G$ contains only one node, then $G$ will be discovered. Assume that the theorem holds for any episode $i \operatorname{cl}\left(G^{\prime}\right)$, where $G^{\prime}$ is a subepisode of $G$. If $G$ has derivable nodes or edges, then by Lemma 7 there exists an episode $G^{\prime} \prec G$ such that $H=i c l(G)=i c l\left(G^{\prime}\right)$ and so by the induction assumption $G^{\prime}$ is discovered, and $H$ is outputted. If $G$ has no derivable edges or nodes, then Theorem 11 implies that $G$ is discovered. This completes the proof.

\section{Experiments}

We tested our algorithm ${ }^{4}$ on three text datasets, address, consisting of the inaugural addresses by the presidents of the United States ${ }^{5}$, merged to form a single long sequence, moby, the novel Moby Dick by Herman Melville ${ }^{6}$, and abstract, consisting of the first $739 \mathrm{NSF}$ award abstracts from $1990^{7}$, also merged into one long sequence. We processed all three sequences using the Porter Stemmer ${ }^{8}$ and removed the stop words. The characteristics of datasets are given in Table 2.

In the implementation an episode graph was implemented using sparse notation: the neighbourhood of a node was presented as a list of edges. To ensure efficient scanning, the sequence was implemented as a set of linked lists, one for each symbol. The experiments were conducted on a computer with an AMD Athlon 64 processor and 2GB memory. The code was compiled with $\mathrm{G}++$ 4.3.4.

We used a window of size 15 for all our experiments and varied the frequency threshold $\sigma$. The main goal of our experiments was to demonstrate how we tackle

\footnotetext{
4 The $\mathrm{C}++$ implementation is given at http://adrem.ua.ac.be/implementations/

5 taken from http://www. bartleby.com/124/pres68

6 taken from http://www.gutenberg.org/etext/15

7 taken from http://kdd.ics.uci.edu/databases/nsfabs/nsfawards.html

8 http://tartarus.org/ martin/PorterStemmer/
} 


\begin{tabular}{lrr}
\hline Sequence & Size & $|\Sigma|$ \\
\hline moby & 105719 & 10277 \\
abstract & 67828 & 6718 \\
address & 62066 & 5295 \\
\hline
\end{tabular}

Table 2 Characteristics of the sequences. The first column contains the size of the sequence, and the second column the number of unique symbols in the sequence.

the problem of pattern explosion. Tables 3 and 4 show how the total number of frequent episodes compared with the identified $i$-closed, $e$-closed and $f$-closed episodes we discovered in the three datasets, using the fixed-window frequency and the disjointwindow frequency, respectively. The results show that while the reduction is relatively small for large thresholds, its benefits are clearly visible as we lower the threshold. The reason for this is that, because of the data characteristics, the major part of the output consists of episodes with a small number of nodes. Such episodes tend to be closed. When the threshold is lowered, episodes with a large number of nodes become frequent which leads to a pattern explosion. In the extreme case, we ran out of memory when discovering all frequent episodes for certain low thresholds while were able to compute the $f$-closed episodes.

\begin{tabular}{lrrrrr}
\hline Dataset & $\sigma$ & $f$-closed & $i$-closed & $e$-closed & frequent \\
\hline address & 200 & 1983 & 1989 & 1989 & 1992 \\
& 100 & 6774 & 6817 & 6820 & 6880 \\
& 50 & 25732 & 26078 & 26212 & 34917 \\
& 30 & 70820 & 72570 & 73328 & 119326 \\
& 20 & 166737 & 192544 & 207153 & out of memory \\
\hline abstract & 500 & 893 & 914 & 916 & 933 \\
& 400 & 1374 & 1436 & 1440 & 1499 \\
& 300 & 2448 & 2802 & 2903 & 4080 \\
& 200 & 6074 & 7374 & 8080 & 98350 \\
& 100 & 26140 & 43020 & 95811 & out of memory \\
\hline moby & 200 & 3389 & 3394 & 3394 & 3395 \\
& 100 & 11018 & 11079 & 11084 & 11127 \\
& 50 & 37551 & 38043 & 38120 & 39182 \\
& 30 & 99937 & 102380 & 103075 & 151115 \\
& 20 & 231563 & 245683 & 253208 & out of memory \\
\hline
\end{tabular}

Table 3 The number of frequent, $i$-closed, $e$-closed and $f$-closed episodes with varying fixedwindow frequency thresholds for the address, abstract and moby datasets, respectively.

To get a more detailed picture we examined the ratio of the number of frequent episodes and the number of $f$-closed episodes, the ratio of the number of $i$-closed episodes and the number of $f$-closed episodes, and the ratio of the number of $e$-closed episodes and the number of $i$-closed episodes, as a function of the number of nodes. The results using the fixed-window frequency are shown in Figure 8. We see that while there is no improvement with small episodes, using closed episodes is essential if we are interested in large episodes. In such a case we were able to reduce the output by several orders of magnitude. For example, in the moby dataset, with a threshold of 30 , there were 24131 frequent episodes of size 6 , of which only 13 were $f$-closed. 


\begin{tabular}{lrrrrr}
\hline Dataset & $\sigma$ & $f$-closed & $i$-closed & $e$-closed & frequent \\
\hline address & 20 & 2264 & 2282 & 2282 & 2291 \\
& 10 & 9984 & 10213 & 10219 & 10396 \\
& 5 & 46902 & 50634 & 50920 & 65139 \\
& 4 & 77853 & 87935 & 89076 & 268675 \\
& 3 & 149851 & 187091 & 195379 & out of memory \\
\hline abstract & 100 & 195 & 195 & 195 & 195 \\
& 50 & 932 & 1000 & 1002 & 1020 \\
& 40 & 1677 & 2053 & 2122 & 2585 \\
& 30 & 3542 & 5482 & 5798 & 20314 \\
& 20 & 9933 & 22945 & 61513 & out of memory \\
\hline moby & 20 & 4076 & 4119 & 4121 & 4121 \\
& 10 & 15930 & 16325 & 16341 & 16468 \\
& 5 & 67180 & 72306 & 72468 & 77701 \\
& 4 & 109572 & 122423 & 122919 & 141940 \\
& 3 & 207031 & 251757 & 158303 & out of memory
\end{tabular}

Table 4 The number of frequent, $i$-closed, $e$-closed and $f$-closed episodes with varying disjointwindow frequency thresholds for the address, abstract and moby datasets, respectively.

Clearly, the number of discovered $i$-closed episodes remains greater than the number of $f$-closed episodes, but does not explode, guaranteeing the feasibility of our algorithm. For example, in the abstract dataset, with a threshold of 200, there were 17587 frequent episodes of size 5 , of which 878 were $i$-closed and $289 f$-closed. Furthermore, we can see that while using only the $e$-closure helps reduce the output significantly, using the $i$-closure gives an even better reduction. For example, in the address dataset, with a threshold of 30 , there were 259 e-closed episodes of size 5 , of which 178 were $i$-closed.

The same ratios using the disjoint-window frequency are shown in Figure 9. Again, we can clearly see the benefits of using $i$-closure, especially on large episodes.

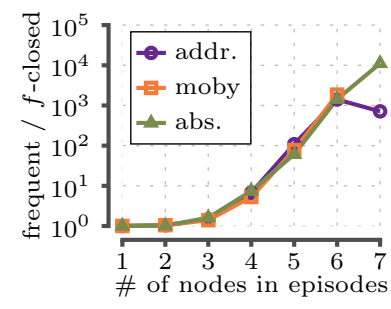

(a) Frequent / $f$-closed

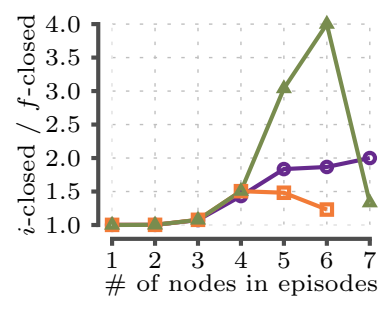

(b) $i$-closed / $f$-closed

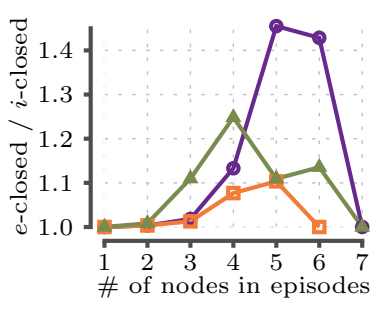

(c) $e$-closed / $i$-closed

Fig. 8 Ratios of episodes as a function of the number of events. $f r_{f}(G)$ was used as frequency with the threshold $\sigma=30$ for address and moby, and $\sigma=200$ for abstract. Note that the $\mathrm{y}$-axis of Figure $8(\mathrm{a})$ is in log-scale.

The difference between the number of $f$-closed and $i$-closed episodes can be explained by the fact that the $i$-closure operator looks at all valid mappings of the episode while the coverage requires only one valid mapping to exist. For example, consider the episode given in Example 1. This episode is $f$-closed with respect to disjoint 


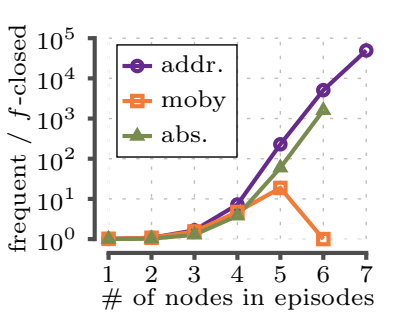

(a) Frequent / f-closed

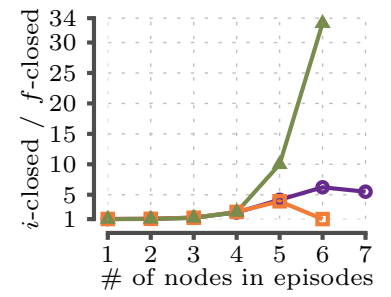

(b) $i$-closed / $f$-closed

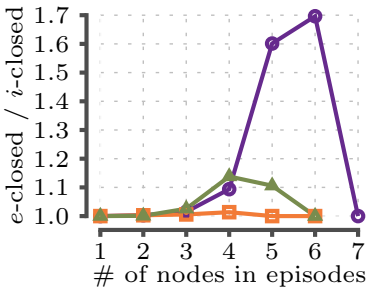

(c) $e$-closed / $i$-closed

Fig. 9 Ratios of episodes as a function of the number of events. $f r_{d}(G)$ was used as frequency with the threshold $\sigma=4$ for address and Moby, and $\sigma=30$ for abstract. Note that the y-axis of Figure $9(\mathrm{a})$ is in log-scale.

windows, and occurs in 10 windows. A subepisode

$$
\text { chief } \rightarrow \text { justic vice president }
$$

is $i$-closed but not $f$-closed. The reason for this is that several speeches contain a line 'vice president ... chief justice ... vice president'. Hence, we can construct a valid mapping for the order: president, chief, justice, vice. Consequently, we cannot add an edge from vice to president. However, for all such mappings we can construct an alternative mapping satisfying the episode in Example 1. Thus the support of both episodes will be the same and we can delete the subepisode from the output.

To complete our analysis, we present a comparison of the number of serial, parallel and general episodes we found. We compare the number of $f$-closed episodes to the overall number of frequent episodes, to illustrate how much the output has been reduced by using closed episodes. The results are shown in Tables 5 and 6 for fixed-window and disjoint-window frequency, respectively. To avoid double counting, we consider singletons to be parallel episodes, while neither serial nor parallel episodes are included in the general episodes total. As expected, the number of serial and parallel episodes does not change much, as most of them are closed. For a serial episode not to be closed, we would need to find an episode consisting of more nodes, yet having the same frequency, which is not often the case. A parallel episode is not closed if a partial order can be imposed on its nodes, without a decline in frequency - again, this is not often the case. However, as has been pointed out in Figure 1 in the introduction, a single frequent serial episode results in an explosion of the number of discovered general episodes. The results demonstrate that the output of general episodes has indeed been greatly reduced. For example, using a fixed-window frequency threshold of 200 on the abstract dataset, we discovered 93813 frequent general episodes, of which only 2247 were $f$-closed.

The runtimes of our experiments varied between a few seconds and 3 minutes for the largest experiments. However, with low thresholds, our algorithm for finding closed episodes ran faster than the algorithm for finding all frequent episodes, and at the very lowest thresholds, our algorithm produced results, while the frequent-episodes algorithm ran out of memory. This demonstrates the infeasibility of approaching the problem by first generating all frequent episodes, and then pruning the non-closed ones. The $i$-closed episodes are the necessary intermediate step. 


\begin{tabular}{lrrrrrrr}
\hline \multirow{2}{*}{ Dataset } & $\sigma$ & $\begin{array}{r}\text { serial } \\
f \text {-closed }\end{array}$ & $\begin{array}{r}\text { serial } \\
\text { frequent }\end{array}$ & $\begin{array}{r}\text { parallel } \\
f \text {-closed }\end{array}$ & $\begin{array}{r}\text { parallel } \\
\text { frequent }\end{array}$ & $\begin{array}{r}\text { general } \\
f \text {-closed }\end{array}$ & $\begin{array}{r}\text { general } \\
\text { frequent }\end{array}$ \\
\hline address & 200 & 293 & 293 & 1670 & 1674 & 20 & 25 \\
& 100 & 1739 & 1742 & 4846 & 4878 & 189 & 260 \\
& 50 & 8436 & 8494 & 15526 & 16068 & 1770 & 10355 \\
& 30 & 24620 & 24973 & 36593 & 41055 & 9607 & 53298 \\
& 20 & 61254 & $\mathrm{~N} / \mathrm{A}$ & 67658 & $\mathrm{~N} / \mathrm{A}$ & 37825 & $\mathrm{~N} / \mathrm{A}$ \\
\hline abstract & 500 & 116 & 116 & 667 & 669 & 110 & 148 \\
& 400 & 206 & 208 & 939 & 942 & 229 & 349 \\
& 300 & 433 & 448 & 1435 & 1471 & 580 & 2161 \\
& 200 & 1124 & 1353 & 2703 & 3184 & 2247 & 93813 \\
& 100 & 4597 & $\mathrm{~N} / \mathrm{A}$ & 7854 & $\mathrm{~N} / \mathrm{A}$ & 13689 & $\mathrm{~N} / \mathrm{A}$ \\
\hline moby & 200 & 594 & 594 & 2772 & 2776 & 23 & 25 \\
& 100 & 2992 & 2997 & 7749 & 7788 & 277 & 342 \\
& 50 & 12529 & 12583 & 22615 & 23192 & 2407 & 3407 \\
& 30 & 34469 & 34915 & 52481 & 58021 & 12987 & 58179 \\
& 20 & 85112 & $\mathrm{~N} / \mathrm{A}$ & 96675 & $\mathrm{~N} / \mathrm{A}$ & 49866 & $\mathrm{~N} / \mathrm{A}$ \\
\hline
\end{tabular}

Table 5 The number of $f$-closed and frequent serial, parallel and general episodes, with varying fixed-window frequency thresholds for the address, abstract and moby datasets, respectively. Singletons are classified as parallel episodes, and general episodes do not include the serial and parallel episodes.

\begin{tabular}{lrrrrrrr}
\hline \multirow{2}{*}{ Dataset } & $\sigma$ & $\begin{array}{r}\text { serial } \\
f \text {-closed }\end{array}$ & $\begin{array}{r}\text { serial } \\
\text { frequent }\end{array}$ & $\begin{array}{r}\text { parallel } \\
f \text {-closed }\end{array}$ & $\begin{array}{r}\text { parallel } \\
\text { frequent }\end{array}$ & $\begin{array}{r}\text { general } \\
f \text {-closed }\end{array}$ & $\begin{array}{r}\text { general } \\
\text { frequent }\end{array}$ \\
\hline address & 20 & 479 & 479 & 1744 & 1753 & 41 & 59 \\
& 10 & 3004 & 3012 & 6325 & 6468 & 655 & 916 \\
& 5 & 15318 & 15557 & 24038 & 27069 & 7546 & 22513 \\
& 4 & 25532 & 26372 & 36116 & 45108 & 16205 & 197195 \\
& 3 & 49859 & $\mathrm{~N} / \mathrm{A}$ & 57293 & $\mathrm{~N} / \mathrm{A}$ & 42699 & $\mathrm{~N} / \mathrm{A}$ \\
\hline abstract & 100 & 20 & 20 & 160 & 160 & 15 & 15 \\
& 50 & 164 & 166 & 565 & 573 & 203 & 281 \\
& 40 & 300 & 314 & 892 & 924 & 485 & 1347 \\
& 30 & 631 & 708 & 1479 & 1660 & 1432 & 17946 \\
& 20 & 1638 & $\mathrm{~N} / \mathrm{A}$ & 2920 & $\mathrm{~N} / \mathrm{A}$ & 5375 & $\mathrm{~N} / \mathrm{A}$ \\
\hline moby & 20 & 1010 & 1014 & 2983 & 3004 & 83 & 103 \\
& 10 & 5075 & 5099 & 9853 & 10035 & 1002 & 1334 \\
& 5 & 22016 & 22304 & 34070 & 37920 & 11094 & 17477 \\
& 4 & 35950 & 36807 & 50545 & 61704 & 23077 & 43429 \\
& 3 & 69048 & $\mathrm{~N} / \mathrm{A}$ & 79944 & $\mathrm{~N} / \mathrm{A}$ & 58039 & $\mathrm{~N} / \mathrm{A}$ \\
\hline
\end{tabular}

Table 6 The number of $f$-closed and frequent serial, parallel and general episodes, with varying disjoint-window frequency thresholds for the address, abstract and moby datasets, respectively. Singletons are classified as parallel episodes, and general episodes do not include the serial and parallel episodes.

\section{Related Work}

Searching for frequent patterns in data is a very common data mining problem. The first attempt at discovering sequential patterns was made by Wang et al. [19]. There, the dataset consists of a number of sequences, and a pattern is considered interesting if it is long enough and can be found in a sufficient number of sequences. The method proposed in this paper, however, was not guaranteed to discover all interesting patterns, but a 
complete solution to a more general problem (dropping the pattern length constraint) was later provided by Agrawal and Srikant [2] using an ApRIORI-style algorithm [1].

It has been argued that not all discovered patterns are of interest to the user, and some research has gone into outputting only closed sequential patterns, where a sequence is considered closed if it is not properly contained in any other sequence which has the same frequency. Yan et al. [21], Tzvetkov et al. [18], and Wang and Han [20] proposed methods for mining such closed patterns, while Garriga [5] further reduced the output by post-processing it and representing the patterns using partial orders. Despite their name, the patterns discovered by Garriga are different from the traditional episodes. A sequence covers an episode if every node of the DAG can be mapped to a symbol such that the order is respected, whereas a partial order discovered by Garriga is covered by a sequence if all paths in the DAG occur in the sequence; however, a single event in a sequence can be mapped to multiple nodes.

In another attempt to trim the output, Garofalakis et al. [7] proposed a family of algorithms called SPIRIT which allow the user to define regular expressions that specify the language that the discovered patterns must belong to.

Looking for frequent episodes in a single event sequence was first proposed by Mannila et al. [12]. The WINEPI algorithm finds all episodes that occur in a sufficient number of windows of fixed length. The frequency of an episode is defined as the fraction of all fixed-width sliding windows in which the episode occurs. The user is required to choose the width of the window and a frequency threshold. Specific algorithms are given for the case of parallel and serial episodes. However, no algorithm for detecting general episodes (DAGs) is provided.

The same paper proposes the MinePI method, where the interestingness of an episode is measured by the number of minimal windows that contain it. As was shown by Tatti [16], Minepi fails due to an error in its definition. Zhou et al. [22] proposed mining closed serial episodes based on the MiNEPI method, without solving this error. Laxman et al. introduced a monotonic measure as the maximal number of nonoverlapping occurrences of the episode [11].

Pei et al. [15] considered a restricted version of our problem setup. In their setup, items are allowed to occur only once in a window (string in their terminology). This means that the discovered episodes can contain only one occurrence of each item. This restriction allows them to easily construct closed episodes. Our setup is more general since we do not restrict the number of occurrences of a symbol in the window and the miner introduced by Pei cannot be adapted to our problem setting since the restriction imposed by the authors plays a vital part in their algorithm.

Garriga [4] pointed out that WINEPI suffers from bias against longer episodes, and proposed solving this by increasing the window length proportionally to the episode length. However, as was pointed out by Méger and Rigotti [13], the algorithm given in this paper contained an error.

An attempt to define frequency without using any windows has been made by Calders et al. [3] where the authors define an interestingness measure of an itemset in a stream to be the frequency starting from a point in time that maximizes it. However, this method is defined only for itemsets, or parallel episodes, and not for general episodes. Cule et al. [6] proposed a method that uses neither a window of fixed size, nor minimal occurrences, and an interestingness measure is defined as a combination of the cohesion and the frequency of an episode - again, only for parallel episodes. Tatti [16] and Gwadera et al. [8,9] define an episode as interesting if its occurrences deviate from expectations. 
Finally, an extensive overview of temporal data mining has been made by Laxman and Sastry [10].

\section{Conclusions}

In this paper, we tackled the problem of pattern explosion when mining frequent episodes in an event sequence. In such a setting, much of the output is redundant, as many episodes have the same frequency as some other, more specific, episodes. We therefore output only closed episodes, for which this is not the case. Further redundancy is found in the fact that some episodes can be represented in more than one way. We solve this problem by restricting ourselves to strict, transitively closed episodes.

Defining frequency-closed episodes created new problems, as, unlike in some other settings, a non-closed frequent episode can have more than one closure. To solve this, we defined a closure operator based on instances. This closure does not suffer from the same problems that occur with the closure based on frequency. Unlike the closure based on frequency, an episode will always have only one instance-closure.

We further proved that every $f$-closed episode must also be $i$-closed. Based on this, we developed an algorithm that efficiently identifies $i$-closed episodes, as well as $f$-closed episodes, in a post-processing step. Experiments have confirmed that the reduction in output is considerable, and essential for large episodes, where we reduced the output by several orders of magnitude. Moreover, thanks to introducing $i$-closed episodes, we can now produce output for thresholds at which finding all frequent episodes is infeasible.

\section{Acknowledgments}

Nikolaj Tatti is supported by a Post-doctoral Fellowship of the Research Foundation Flanders (FWO).

\section{References}

1. Rakesh Agrawal and Ramakrishnan Srikant. Fast algorithms for mining association rules. In Proceedings of the 20th International Conference on Very Large Data Bases (VLDB 1994), pages 487-499, 1994.

2. Rakesh Agrawal and Ramakrishnan Srikant. Mining sequential patterns. 11th International Conference on Data Engineering (ICDE 1995), 0:3-14, 1995.

3. Toon Calders, Nele Dexters, and Bart Goethals. Mining frequent itemsets in a stream. In Proceedings of the 7th IEEE International Conference on Data Mining (ICDM 2007), pages 83-92, 2007.

4. Gemma Casas-Garriga. Discovering unbounded episodes in sequential data. In Knowledge Discovery in Databases: PKDD 2003, 7th European Conference on Principles and Practice of Knowledge Discovery in Databases, pages 83-94, 2003.

5. Gemma Casas-Garriga. Summarizing sequential data with closed partial orders. In Proceedings of the SIAM International Conference on Data Mining (SDM 2005), pages 380391, 2005.

6. Boris Cule, Bart Goethals, and Céline Robardet. A new constraint for mining sets in sequences. In Proceedings of the SIAM International Conference on Data Mining (SDM 2009), pages 317-328, 2009.

7. Minos Garofalakis, Rajeev Rastogi, and Kyuseok Shim. Mining sequential patterns with regular expression constraints. IEEE Transactions on Knowledge and Data Engineering, 14(3):530-552, 2002. 
8. Robert Gwadera, Mikhail J. Atallah, and Wojciech Szpankowski. Markov models for identification of significant episodes. In Proceedings of the SIAM International Conference on Data Mining (SDM 2005), pages 404-414, 2005.

9. Robert Gwadera, Mikhail J. Atallah, and Wojciech Szpankowski. Reliable detection of episodes in event sequences. Knowledge and Information Systems, 7(4):415-437, 2005.

10. Srivatsan Laxman and P. S. Sastry. A survey of temporal data mining. SADHANA, Academy Proceedings in Engineering Sciences, 31(2):173-198, 2006.

11. Srivatsan Laxman, P. S. Sastry, and K. P. Unnikrishnan. A fast algorithm for finding frequent episodes in event streams. In Proceedings of the 13th ACM SIGKDD International Conference on Knowledge discovery and data mining (KDD 2007), pages 410-419, 2007.

12. Heikki Mannila, Hannu Toivonen, and A. Inkeri Verkamo. Discovery of frequent episodes in event sequences. Data Mining and Knowledge Discovery, 1(3):259-289, 1997.

13. Nicolas Méger and Christophe Rigotti. Constraint-based mining of episode rules and optimal window sizes. In Knowledge Discovery in Databases: PKDD 2004, 8th European Conference on Principles and Practice of Knowledge Discovery in Databases, pages 313$324,2004$.

14. Nicolas Pasquier, Yves Bastide, Rafik Taouil, and Lotfi Lakhal. Discovering frequent closed itemsets for association rules. In ICDT '99: Proceedings of the 7th International Conference on Database Theory, pages 398-416, 1999.

15. Jian Pei, Haixun Wang, Jian Liu, Ke Wang, Jianyong Wang, and Philip S. Yu. Discovering frequent closed partial orders from strings. IEEE Transactions on Knowledge and Data Engineering, 18(11):1467-1481, 2006.

16. Nikolaj Tatti. Significance of episodes based on minimal windows. In Proceedings of the 9th IEEE International Conference on Data Mining (ICDM 2009), pages 513-522, 2009.

17. Nikolaj Tatti and Boris Cule. Mining closed strict episodes. In Proceedings of the 10th IEEE International Conference on Data Mining (ICDM 2010), pages 501-510, 2010.

18. Petre Tzvetkov, Xifeng Yan, and Jiawei Han. Tsp: Mining top-k closed sequential patterns. In Proceedings of the 3rd IEEE International Conference on Data Mining (ICDM 2003), pages $347-354,2003$.

19. Jason Tsong-Li Wang, Gung-Wei Chirn, Thomas G. Marr, Bruce Shapiro, Dennis Shasha, and Kaizhong Zhang. Combinatorial pattern discovery for scientific data: some preliminary results. ACM SIGMOD Record, 23(2):115-125, 1994.

20. Jianyong Wang and Jiawei Han. Bide: Efficient mining of frequent closed sequences. 20th International Conference on Data Engineering (ICDE 2004), 0:79, 2004.

21. Xifeng Yan, Jiawei Han, and Ramin Afshar. Clospan: Mining closed sequential patterns in large datasets. In Proceedings of the SIAM International Conference on Data Mining (SDM 2003), pages 166-177, 2003.

22. Wenzhi Zhou, Hongyan Liu, and Hong Cheng. Mining closed episodes from event sequences efficiently. In Proceedings of the 14th Pacific-Asia Conference on Knowledge Discovery and Data Mining(1), pages 310-318, 2010. 